\title{
Medium-induced jet evolution: wave turbulence and energy loss
}

\author{
Leonard Fister and Edmond lancu \\ Institut de Physique Théorique de Saclay, \\ F-91191 Gif-sur-Yvette, France \\ E-mail: leonard.fister@cea.fr, edmond.iancu@cea.fr
}

ABSTRACT: We study the gluon cascade generated via successive medium-induced branchings by an energetic parton propagating through a dense QCD medium. We focus on the high-energy regime where the energy $E$ of the leading particle is much larger than the characteristic medium scale $\omega_{c}=\hat{q} L^{2} / 2$, with $\hat{q}$ the jet quenching parameter and $L$ the distance travelled through the medium. In this regime the leading particle loses only a small fraction $\sim \alpha_{s}\left(\omega_{c} / E\right)$ of its energy and can be treated as a steady source of radiation for gluons with energies $\omega \leq \omega_{c}$. For this effective problem with a source, we obtain exact analytic solutions for the gluon spectrum and the energy flux. These solutions exhibit wave turbulence: the basic physical process is a continuing fragmentation which is 'quasidemocratic' (i.e. quasi-local in energy) and which provides an energy transfer from the source to the medium at a rate (the energy flux $\mathcal{F}$ ) which is quasi-independent of $\omega$. The locality of the branching process implies a spectrum of the Kolmogorov-Obukhov type, i.e. a power-law spectrum which is a fixed point of the branching process and whose strength is proportional to the energy flux: $D(\omega) \sim \mathcal{F} / \sqrt{\omega}$ for $\omega \ll \omega_{c}$. Via this turbulent flow, the gluon cascade loses towards the medium an energy $\Delta E \sim \alpha_{s}^{2} \omega_{c}$, which is independent of the initial energy $E$ of the leading particle and of the details of the thermalization mechanism at the low-energy end of the cascade. This energy is carried away by very soft gluons, which propagate at very large angles with respect to the jet axis. Our predictions for the value of $\Delta E$ and for its angular distribution appear to agree quite well, qualitatively and even semi-quantitatively, with the phenomenology of di-jet asymmetry in nucleus-nucleus collisions at the LHC.

KeYwords: Quark-Gluon Plasma, QCD, Resummation, Parton Model

ARXIV EPRINT: 1409.2010 


\section{Contents}

1 Introduction 1

2 The low-energy regime $\quad 6$

2.1 The rate equation 6

2.2 The spectrum and the flow energy 8

$\begin{array}{lll}2.3 \text { Energy flux, turbulence, and thermalization } & 11\end{array}$

3 The high-energy regime $\quad 14$

$\begin{array}{ll}3.1 \text { The coupled rate equations } & 15\end{array}$

$\begin{array}{lll}3.2 & \text { The radiation spectrum } & 18\end{array}$

$\begin{array}{ll}3.3 & \text { The energy flux } \\ 3.4 & 22\end{array}$

3.4 The energy flux revisited: democratic branchings 26

4 Physical discussion: energy loss at large angles $\quad 30$

$\begin{array}{ll}\text { A Perturbation theory for the rate equation } & 37\end{array}$

\section{Introduction}

The experimental observation of the phenomenon known as 'di-jet asymmetry' in $\mathrm{Pb}+\mathrm{Pb}$ collisions at the LHC [1-8] has triggered intense theoretical efforts [9-21], aiming at understanding the evolution of an energetic jet propagating through a dense QCD medium, such as a quark-gluon plasma. An important conclusion of these studies is that the interactions between the partons composing the jet (the leading particle and the products of its fragmentation) and the constituents of the medium lead to a specific in-medium fragmentation pattern, in which the main role is played by relatively soft gluon emissions, with energies $\omega$ much smaller than the characteristic medium scale $\omega_{c}=\hat{q} L^{2} / 2$. Such soft gluons are abundantly radiated inside the medium because the associated formation time $t_{\mathrm{br}}(\omega) \sim \sqrt{\omega / \hat{q}}$ (the typical duration of a branching process triggered by multiple scattering) is much smaller than the distance $L$ traveled by the leading particle through the medium. In the previous formulæ, $\hat{q}$ is the 'jet quenching parameter', a transport coefficient which measures the dispersion in transverse momentum accumulated per unit time by an energetic parton which undergoes multiple scattering in the medium. This conclusion has far reaching consequences, in particular for the phenomenology of di-jet asymmetry.

The soft gluons can be easily deviated towards large angles by rescattering in the medium. Therefore, their abundant production via jet fragmentation may explain the significant transport of energy to large angles with respect to the jet axis - the hallmark of di-jet asymmetry. Also, the successive emissions of soft gluons are well separated from 
each other and can be described as a classical, probabilistic, branching process. Indeed, the quantum coherence effects and the associated interference phenomena are efficiently washed out by rescattering in the medium [9-11]. The loss of color coherence occurs on a time scale comparable to that of the branching process. Hence, even gluons which emerge from the same splitting vertex can be viewed as propagating independently of each other [14].

Based on such considerations, one has been able to derive a classical effective theory for the gluon cascade generated via successive medium-induced gluon branchings $[16,17]$ (see also refs. [22-24] for earlier, related, studies). This is a stochastic theory for a Markovian process in which the branching rate is given by the BDMPSZ spectrum ${ }^{1}$ [25-29] for a single medium-induced emission. The branching probability corresponding to a distance $L$ is parametrically of order $\bar{\alpha}\left[L / t_{\mathrm{br}}(\omega)\right]$, with $\bar{\alpha} \equiv \alpha_{s} N_{c} / \pi$. For the relatively hard emissions $\left(\omega \sim \omega_{c}\right)$ this probability is small, of $\mathcal{O}(\bar{\alpha})$. On the other hand, for $\omega \lesssim \omega_{s} \equiv \bar{\alpha}^{2} \omega_{c}$ it becomes of order one, meaning that the branching dynamics becomes non-perturbative. As we shall see, this 'soft' scale $\omega_{s}$ is truly semi-hard (in the ballpark of a few $\mathrm{GeV}$ ), so there is a significant region in phase-space where naive perturbation theory breaks down. The effective theory put forward in refs. $[16,17]$ allows one to deal with such non-perturbative aspects, by resumming soft multiple branchings to all orders.

The original analysis in [16] demonstrated that the non-perturbative dynamics associated with multiple branchings has a remarkable consequence: it leads to wave turbulence [30,31]. The leading particle, whose initial energy $E$ is typically much larger than the non-perturbative scale $\omega_{s}$, promptly and abundantly radiates soft gluons with energies $\omega \lesssim \omega_{s}$ and thus loses an energy amount $\Delta E \sim \omega_{s}$ event by event. After being emitted, these soft primary gluons keep on branching into even softer gluons, and their subsequent branchings are quasi-democratic: the two daughter gluons produced by a typical splitting have comparable energies. ${ }^{2}$ This democratic nature of the branching is the key ingredient for turbulence. It leads to a power-law spectrum $D(\omega) \propto 1 / \sqrt{\omega}$, which emerges as the Kolmogorov-Zakharov (KZ) fixed point $[30,31]$ of the respective rate equation, and to an energy flux which is independent of $\omega$ - the turbulent flow. An energy flux uniform in $\omega$ means that the energy flows from the high-energy end to the low-energy end of the cascade, without accumulating at any intermediate value of $\omega$. For an ideal cascade the branching law remains unchanged down to arbitrary small values of $\omega$ and thus the energy accumulates into a condensate at $\omega=0$. In practice, we expect the branching process to be modified when the gluon energies become comparable to the medium 'temperature' $T$ (the typical energy of the medium constituents): the soft gluons with $\omega \sim T$ 'thermalize', meaning that they transfer their energy towards the medium. In that case, the rate for energy loss is controlled by the turbulent flow and thus independent of the details of the thermalization mechanism. This universality is a well known feature of turbulence [30, 31].

The crucial property of the turbulent flow for our present purposes is the fact that it can transfer a significant fraction of the original energy of the leading particle towards

\footnotetext{
'BDMPSZ' stands for Baier, Dokshitzer, Mueller, Peigné, Schiff, and Zakharov.

${ }^{2}$ It is interesting to notice that a similar branching process occurs in a different physical context, namely the thermalization of the quark-gluon plasma produced in the intermediate stages of a ultrarelativistic heavy ion collision: during the late stages of the 'bottom-up' scenario [22], the hard particles lose energy towards the surrounding thermal bath via soft radiation giving rise to quasi-democratic cascades [32].
} 
arbitrarily soft quanta. This fact is highly non-trivial, as it can be best appreciated by comparing with the traditional parton cascade in perturbative QCD: the DGLAP cascade for a jet fragmenting in the vacuum. In the DGLAP cascade, the typical splittings are very asymmetric, due to the 'infrared' $(\omega \rightarrow 0)$ singularity of bremsstrahlung, and lead to a rapid rise in the number of gluons with small values of the energy fraction $x \equiv \omega / E$. Yet, the total energy carried by these 'wee' gluons with $x \ll 1$ is very small: the energy fraction contained in the region of the spectrum at $x<x_{0}$ vanishes as a power of $x_{0}$ when $x_{0} \rightarrow 0$. Most of the original energy remains in the few partons with larger values of $x$. This is due to the fact that, after a very asymmetric splitting, the parent parton preserves most of its original energy.

By contrast, for the in-medium cascade, where splittings are quasi-democratic, the energy contained in the bins of the spectrum at $x<x_{0}$ is only a part of the total energy which has 'fallen' below $x_{0}$. The other part is the energy carried by the turbulent flow, which ends up at arbitrarily low values of $x$ and hence is independent of $x_{0}$. In the presence of a thermalization mechanism at $\omega \sim T$, this argument remains valid so long as $x_{0} \geq x_{\mathrm{th}}$, with $x_{\mathrm{th}} \equiv T / E$. In practice, this value $x_{\mathrm{th}} \sim 10^{-2}$ is quite small, so most of the energy lost by the gluon cascade towards the medium is associated with the turbulent flow, and not with the region of the spectrum at $x \lesssim x_{\mathrm{th}}$. Depending upon the size $L$ of the medium, the whole initial energy $E$ can be lost in this way.

Soft gluons propagate at large angles $\theta$ with respect to the jet axis: $\theta \sim k_{\perp} / \omega$, where $k_{\perp}$ is the typical transverse momentum acquired by the gluon via rescattering in the medium; roughly, $k_{\perp}^{2} \sim Q_{L}^{2} \equiv \hat{q} L$. So, the ability of the medium-induced cascade to abundantly produce soft gluons provides a natural explanation for the main feature of di-jet asymmetry: the fact that the energy difference between the trigger jet and the away jet is carried by many soft $(p \lesssim 2 \mathrm{GeV})$ hadrons propagating at large angles $(\theta \gtrsim 0.8)$ with respect to the axis of the away jet [2]. This qualitative explanation has been originally proposed in [16] and further developed in refs. [17-20]. However, these previous studies were not fully conclusive, as they did not explicitly consider the kinematical regime which is pertinent for di-jet asymmetry. Namely, they focused on the 'low-energy' regime where the energy $E$ of the leading particle (LP) is smaller than the medium scale $\omega_{c}$. Albeit the value of $\omega_{c}$ is not precisely known from first principles, its current phenomenological estimates are well below the energy $E \gtrsim 100 \mathrm{GeV}$ of the trigger jet in the experimental measurements of di-jet asymmetry (see the discussion in section 4). It is our main objective in this paper to provide a thorough analysis of the high-energy regime at $E \gg \omega_{c}$, including its implications for the phenomenology.

As compared to the low-energy case, the main new feature emerging at high energy is a gap in the spectrum, which separates between the leading-particle peak at $\omega \simeq E$ and a continuum at $\omega \leq \omega_{c}$ representing the radiation. To understand this structure, we recall that, when $E>\omega_{c}$, the energy loss by the LP is controlled by hard but rare emissions with $\omega \sim \omega_{c}$, leading to an average energy $\operatorname{loss}^{3}\langle\Delta E\rangle \sim \bar{\alpha} \omega_{c}$ [25-28]. Accordingly, a very energetic particle with $E \gg \omega_{c}$ loses only a tiny fraction $\bar{\alpha}\left(\omega_{c} / E\right) \ll 1$ of its original energy

\footnotetext{
${ }^{3}$ The $\bar{\alpha}$ factor in $\langle\Delta E\rangle$ appears because an emission with $\omega \sim \omega_{c}$ occurs with a low probability of $\mathcal{O}(\bar{\alpha})$.
} 
and hence emerges from the medium with an energy $E^{\prime} \sim E$, which is much larger than the maximal energy $\omega_{c}$ of its radiation. This explains the gap in the spectrum (see section 3 for details). The detailed structure of the LP peak is unimportant for the di-jet asymmetry: the energy carried by the LP is strongly collimated around the jet axis, within an angle $\theta_{\mathrm{LP}} \sim Q_{L} / E \ll 1$, which is much smaller than the angular opening $\theta_{J}$ of the experimentally defined 'jet'. (Some typical values are $Q_{L}=2 \mathrm{GeV}$ and $E=100 \mathrm{GeV}$, hence $\theta_{\mathrm{LP}} \sim 0.02$, whereas $\theta_{J} \geq 0.2$.) This is in agreement with the experimental observation $[1,2]$ that the azimuthal distribution of di-jets in $\mathrm{Pb}+\mathrm{Pb}$ collisions is as narrowly peaked at $\Delta \phi=\pi$ as the corresponding distribution in $\mathrm{p}+\mathrm{p}$ collisions. What actually matters for the phenomenology of di-jet asymmetry is the continuum part of the spectrum at $\omega<\omega_{c}$ and, especially, its very soft sector at $\omega \lesssim \omega_{s}=\bar{\alpha}^{2} \omega_{c}$, which controls the energy loss at large angles.

In view of the above, our subsequent analysis focuses on the radiation part of the spectrum at $x \leq x_{c}$, where we recall that $x=\omega / E$ and $x_{c}=\omega_{c} / E \ll 1$. For that purpose, the LP can be treated as a steady source of radiation for soft gluons with energy fractions $x \leq x_{c}$. For this effective problem with a source, we are able to construct exact solutions for the gluon spectrum $D(x, t)$ at any time $t \leq L$, and also for the energy flux $\mathcal{F}(x, t)$ (the rate for energy transfer throughout the cascade; see section 2 for a precise definition). This energy flux, and more precisely its 'flow' limit $\mathcal{F}_{\text {flow }}(t) \equiv \mathcal{F}(x=0, t)$, is the most interesting quantity in the present context, since it controls the energy transfer from the gluon cascade to the medium.

A non-zero 'flow' component in the energy flux is the main signature of turbulence [30, 31] (e.g., there is no such a component for the DGLAP cascade). An important property of turbulence, which follows from the locality of the branchings, is the celebrated KolmogorovObukhov relation between the spectrum and the energy flux. A priori, our problem differs from the familiar turbulence set-up via its explicit time-dependence: the source acts only up to a finite time $t_{\max }=L$, which moreover is quite small, in the sense that $\hat{q} L^{2} \ll E$. (In that sense, our problem corresponds to transient phenomena in the context of turbulence, for which very little is known in general.) Notwithstanding, we shall demonstrate that a time-dependent generalization of the Kolmogorov-Obukhov relation holds for the problem at hand: the gluon spectrum at $x \ll x_{c}$ is fully determined by the turbulent flow $\mathcal{F}_{\text {flow }}(t)$ together with the characteristic KZ scaling. Namely, we shall find $D(x, t) \propto \mathcal{F}_{\text {flow }}(t) / \sqrt{x}$, where the proportionality constant is under control too.

The energy transferred by the gluon cascade to the medium can be identified with the energy $\Delta E_{\text {flow }}$ carried away by the flow, i.e. the time integral of $\mathcal{F}_{\text {flow }}(t)$ between $t=0$ and $t=L$. For the high-energy regime under consideration, this quantity turns out to be independent of the original energy $E$ of the LP and to have a transparent physical interpretation: ${ }^{4} \Delta E_{\text {flow }} \simeq v \omega_{s}$, where $\omega_{s}=\bar{\alpha}^{2} \omega_{c}$ and $v \simeq 4.96$ is a pure number which can be interpreted as the average number of soft primary emissions with energies $\omega \sim \omega_{s}$. Such soft gluons are radiated by the LP with probability of order one and they subsequently transfer their energy towards the medium via quasi-democratic branchings. A typical gluon

\footnotetext{
${ }^{4}$ This estimate for $\Delta E_{\text {flow }}$ holds to leading order in $\bar{\alpha}$; see eq. (4.4) and the plots in section 4 for more accurate results.
} 


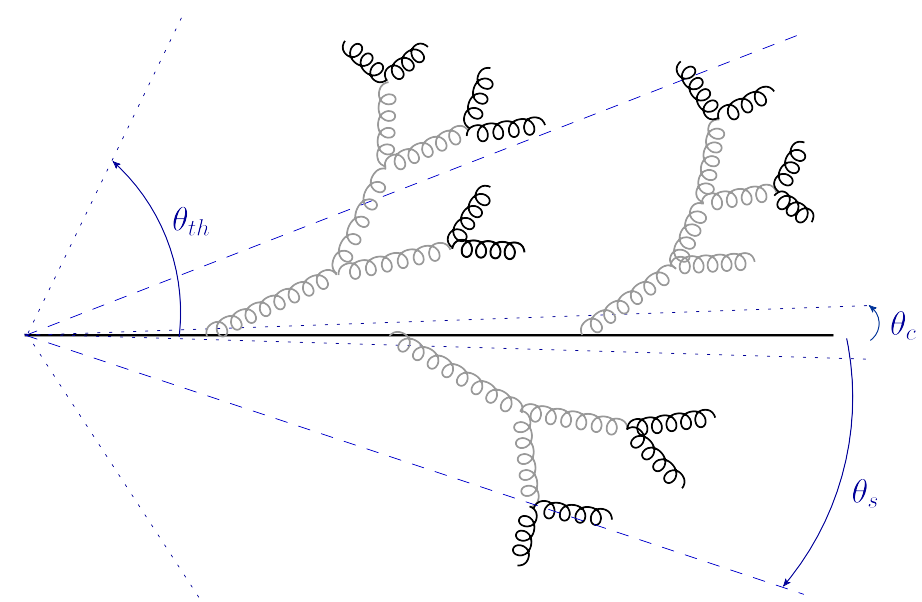

Figure 1. A typical gluon cascade as generated via medium-induced gluon branchings. The small angle $\theta_{c} \simeq Q_{L} / \omega_{c}$ is the propagation angle for a relatively hard gluon with energy $\omega \sim \omega_{c}$. Such a hard emission is a rare event and hence is not included in our typical event. All the shown gluons (besides the LP) have soft energies $\omega \lesssim \omega_{s}=\bar{\alpha}^{2} \omega_{c}$, hence their emissions occur with probability of $\mathcal{O}(1)$. The primary gluons are emitted (by the LP) at a typical angle $\theta_{s}=\theta_{c} / \bar{\alpha}^{2}$ and subsequently disappear via branching into even softer gluons. The opaque lines refer to gluons which exist at intermediate stages of the cascade, while the black lines refer to the 'final' gluons, which thermalize and propagate at even larger angles, $\theta \sim \theta_{\mathrm{th}} \gg \theta_{s}$ (see section 4 for details).

cascade is illustrated in figure 1 . Using phenomenologically motivated values for $\hat{q}$ and $L$, we find $\Delta E_{\text {flow }} \simeq 10 \div 20 \mathrm{GeV}$ (see section 4 ). Since carried by very soft gluons, with energies $\omega \sim T \ll \omega_{s}$, this energy propagates at very large angles with respect to the jet axis, at least as large as $\theta_{s} \equiv Q_{L} / \omega_{s} \sim 0.5$. $\left(\theta_{s}\right.$ is the typical propagation angle of the soft primary gluons.) By progressively increasing the jet opening angle $\theta_{0}$ above $\theta_{s}$, one recovers part of the missing energy, but only very slowly: most of this energy lies at even larger angles, $\theta \sim \theta_{\mathrm{th}}>\theta_{s}$ (see figure 1 and section 4 for details). The above predictions the numerical estimate for the energy loss at large angles $\Delta E_{\text {flow }}$ and its extremely weak dependence upon the jet opening angle $\theta_{0}$ - are in good agreement, qualitative and even semi-quantitative, with the phenomenology of di-jet asymmetry at the LHC $[2,4,5,8]$. Whereas this agreement is, of course, encouraging, it must be taken with a grain of salt, because of the limitations inherent in our formalism (for instance, we ignore the parton virtualities, which would provide an additional mechanism for radiation) and also because of the difficulty to relate our theoretical predictions to the actual measurements at the LHC.

Our paper is organized as follows. In section 2, we shall consider the low-energy regime at $E \lesssim \omega_{c}$ as a warm-up. Besides a succinct review of the main results obtained in ref. [16], this section contains some new material, like the explicit calculation of the energy flux and a first discussion of the Kolmogorov-Obukhov relation. Sections 3 and 4 are devoted to the main new problem of interest for us here: the high-energy regime at $E \gg \omega_{c}$. Section 3 presents the main theoretical developments: the justification of the effective problem with a source, the exact, analytic and numerical, solutions for the radiation spectrum at $\omega \leq \omega_{c}$ and for the turbulent flow, the democratic nature of the 
branchings and its physical implications, and the proof of the (time-dependent version of the) Kolmogorov-Obukhov relation for the branching dynamics at hand. Finally, in section 4 we shall discuss some phenomenological consequences of this dynamics for the energy lost by the jet via soft gluons propagating at large angles.

\section{The low-energy regime}

We would like to study the gluon cascade generated via successive medium-induced gluon branchings by an original gluon - the 'leading particle' (LP) - with energy $E$ which propagates through a dense QCD medium along a distance $L$. For simplicity, we assume the LP to be on its mass-shell before it enters the medium, so that there would be no radiation in the absence of the medium. Accordingly, the parton cascades to be considered in what follows include only those quanta which are produced via medium-induced fragmentation, according to the BDMPSZ mechanism (meaning that several soft scatterings in the medium coherently contribute to a single gluon emission [25-29]).

As explained in the Introduction, there are two interesting physical regimes, depending upon the ratio between $E$ and the characteristic medium scale $\omega_{c}=\hat{q} L^{2} / 2$ :

(i) the high energy regime $E \gg \omega_{c}$, where the LP emerges out of the medium with an energy $E^{\prime}$ which is close to its original energy and hence much larger than the maximal energy $\omega_{c}$ of its radiation, so there is a gap in the spectrum;

(ii) the low energy regime $E \lesssim \omega_{c}$, where the LP loses a significant fraction of its energy via medium-induced radiation and can even disappear inside the medium (in the sense of not being distinguishable from its products of fragmentation).

Recalling that $\omega_{c}=\hat{q} L^{2} / 2$, we see that the 'high energy' case can also be viewed as the limit where the in-medium path $L$ is relatively small, whereas the 'low energy' case corresponds to relatively large values of $L$.

In preparation for the analysis of the high energy regime, which is our main interest in this paper, we shall first discuss the low energy case in some detail. This is useful for several reasons. Besides providing a benchmark for the comparison with the highenergy case, the corresponding results at low energy also enter as 'technical ingredients' in the calculations at high energy. So, by introducing them in the present section, we shall considerably streamline the subsequent discussion of the high-energy regime. Moreover, some of the original results to be obtained in this paper, like the 'jet quenching version' of the Kolmogorov-Obukhov relation, apply to both cases (high energy and low energy) and can be more pedagogically introduced in the familiar set-up at low energy.

\subsection{The rate equation}

Let us first introduce the main notations and equations to be used throughout this paper. The basic quantity that we would like to compute is the gluon spectrum integrated over transverse momenta,

$$
D(\omega, t) \equiv \omega \frac{\mathrm{d} N}{\mathrm{~d} \omega}=\int \mathrm{d}^{2} \boldsymbol{k} \omega \frac{\mathrm{d} N}{\mathrm{~d} \omega \mathrm{d}^{2} \boldsymbol{k}}
$$


where $\omega \leq E$ and $\boldsymbol{k}$ denote the energy and, respectively, transverse momentum of a gluon in the cascade, $N$ is the number of gluons and $t$ the evolution time obeying $0 \leq t \leq L$. The function $D(\omega, t)$ describes the energy distribution within the cascade and its evolution with time. For sufficiently soft gluons at least, namely as long as $\omega \ll \omega_{c}$, and to leading order $^{5}$ in $\alpha_{s}$, this evolution can be described as a classical stochastic branching process [14, 16, 17], with the elementary splitting rate determined by the BDMPSZ spectrum [25-29]. Specifically, the differential probability per unit time and per unit $z$ for a gluon with energy $\omega$ to split into two gluons with energy fractions respectively $z$ and $1-z$ is

$$
\frac{\mathrm{d}^{2} \mathcal{P}_{\mathrm{br}}}{\mathrm{d} z \mathrm{~d} t}=\frac{\alpha_{s}}{2 \pi} \frac{P_{g \rightarrow g}(z)}{t_{\mathrm{br}}(z, \omega)}
$$

where $P_{g \rightarrow g}(z)=N_{c}[1-z(1-z)]^{2} / z(1-z)$, with $0<z<1$, is the leading order gluon-gluon splitting function, $N_{c}$ is the number of colors, and $t_{\mathrm{br}}(z, \omega)$ is the typical duration of the branching process:

$$
t_{\mathrm{br}}(z, \omega) \equiv \sqrt{\frac{z(1-z) \omega}{\hat{q}_{\mathrm{eff}}(z)}}, \quad \hat{q}_{\mathrm{eff}}(z) \equiv \hat{q}[1-z(1-z)] .
$$

Note that this branching time depends on both the energy $\omega$ of the parent gluon and the splitting fraction $z$, which is much smaller than $L$ whenever at least one of the two daughter particles, with energies $z \omega$ and respectively $(1-z) \omega$, is soft compared to $\omega_{c}$.

The elementary splitting rate (2.2) together with the requirement of probability conservation completely specifies the structure of the stochastic branching process and, in particular, the evolution equation obeyed by the gluon spectrum. As long as $E<\omega_{c}$, this equation reads

$$
\frac{\partial D(x, \tau)}{\partial \tau}=\bar{\alpha} \int \mathrm{d} z \mathcal{K}(z)\left[\sqrt{\frac{z}{x}} D\left(\frac{x}{z}, \tau\right)-\frac{z}{\sqrt{x}} D(x, \tau)\right]
$$

in convenient notations where $\bar{\alpha} \equiv \alpha_{s} N_{c} / \pi, D(x, \tau) \equiv D(\omega, t)$ with $x \equiv \omega / E \leq 1$ the energy fraction with respect to the leading particle, and

$$
\tau \equiv \sqrt{\frac{\hat{q}}{E}} t=\sqrt{2 x_{c}} \frac{t}{L}, \quad x_{c} \equiv \frac{\omega_{c}}{E},
$$

is the reduced time (the evolution time in dimensionless units). This variable $\tau$ takes values between $\tau=0$ and a maximal value $\tau_{L} \equiv \sqrt{2 x_{c}}=\sqrt{\hat{q} / E} L$, which increases with the medium size $L$, but decreases with the energy $E$ of the LP. Notice that $x_{c}>1$ for the low-energy regime to be discussed in this section. The splitting kernel $\mathcal{K}(z)$ is defined as

$$
\mathcal{K}(z) \equiv \frac{f(z)}{[z(1-z)]^{3 / 2}}=\mathcal{K}(1-z), \quad f(z) \equiv[1-z(1-z)]^{5 / 2} .
$$

\footnotetext{
${ }^{5}$ A class of particularly large radiative corrections, which are enhanced by the double-logarithm $\ln ^{2}(L T)$, can be effectively resummed into the effective dynamics by replacing the 'bare' value of the jet quenching parameter $\hat{q}$ by its renormalized value, as recently computed in refs. [33-37].
} 
It depends only on the splitting fraction $z$ since the corresponding dependence upon the energy fraction $x$ of the leading particle, cf. eq. (2.2), has been explicitly factored out in writing eq. (2.4).

We shall refer to the r.h.s. of eq. (2.4) as the 'branching term' and denote it as $\bar{\alpha} \mathcal{I}[D]$. This is the sum of two terms, which can be recognized as the familiar 'gain' and 'loss' terms characteristic of a branching process. The first term, which is positive and nonlocal in $x$, is the gain term: it describes the rise in the number of gluons at $x$ due to emissions from gluons at larger $x^{\prime}=x / z$. The respective integral over $z$ is restricted to $x<z<1$ by the support of $D(x / z, \tau)$. The second, negative, term, which is local in $x$, represents the loss term and describes the reduction in the number of gluons at $x$ due to their decay into gluons with smaller $x^{\prime}=z x$. Taken separately, the gain term and the loss term in eq. (2.4) have endpoint singularities at $z=1$, but these singularities exactly cancel between the two terms and the overall equation is well defined.

\subsection{The spectrum and the flow energy}

By integrating eq. (2.2) over $\mathrm{d} t$ and over $z$ with some lower cutoff $z_{0} \ll 1$, one obtained an estimate for the probability to emit a gluon with energy larger than $\omega_{0} \equiv z_{0} \omega$ during a time interval $\Delta t$ :

$$
\Delta \mathcal{P}_{\mathrm{br}}\left(\omega_{0}\right) \sim \bar{\alpha} \frac{\Delta t}{t_{\mathrm{br}}\left(\omega_{0}\right)} \sim \bar{\alpha} \sqrt{\frac{\hat{q}}{2 \omega_{0}}} \Delta t
$$

This probability becomes of order one, meaning that multiple branchings start to be important, when $\omega_{0} \lesssim \omega_{s}(\Delta t) \equiv \bar{\alpha}^{2} \hat{q} \Delta t^{2} / 2$. Note that, for any $\Delta t \leq L$, there exists a sufficiently soft sector, at $\omega_{0} \lesssim \omega_{s}(\Delta t)$, where the branching dynamics is non-perturbative. The typical duration of such a soft branching is parametrically small at weak coupling, $t_{\mathrm{br}}(\omega) \sim \bar{\alpha} \Delta t \ll \Delta t$ for $\omega \sim \omega_{s}(\Delta t)$, hence there is enough phase-space for this nonperturbative dynamics to manifest itself. In particular, for $\Delta t=L$, one recovers the 'soft' scale $\omega_{s}$ introduced in section 1: $\omega_{s}(L)=\omega_{s}$.

To study the effects of multiple branchings, one needs a non-perturbative solution to eq. (2.4). Whereas it is straightforward to solve this equation via numerical methods, for the purpose of demonstrating subtle physical phenomena, it is much more convenient to dispose of an analytic solution. Such a solution has been obtained in ref. [16], but for the simplified kernel $\mathcal{K}_{0}(z) \equiv 1 /[z(1-z)]^{3 / 2}$, which is obtained from eq. (2.6) after replacing the slowly varying factor $f(z)$ in the numerator by 1 . This simplified kernel has the same singularities at $z=0$ and $z=1$ as the original kernel $\mathcal{K}(z)$, hence it is expected to have similar physical implications, at least qualitatively. (This will also be checked via numerical simulations later on; see e.g. figure 4.)

For the simplified kernel $\mathcal{K}_{0}(z)$ and the initial condition $D(x, \tau=0)=\delta(x-1)$, corresponding to a single gluon (the 'leading particle' ) carrying all the energy at $\tau=0$, the exact solution reads [16]

$$
D(x, \tau)=\frac{\bar{\alpha} \tau}{\sqrt{x}(1-x)^{3 / 2}} \exp \left\{-\frac{\pi \bar{\alpha}^{2} \tau^{2}}{1-x}\right\}
$$


This is recognized as the product between the BDMPSZ spectrum [25-29] (which is the same as the result of the first iteration of eq. (2.4)),

$$
D_{0}(x, \tau)=\frac{\bar{\alpha} \tau}{\sqrt{x}(1-x)^{3 / 2}},
$$

and a Gaussian factor describing the broadening of the peak associated with the LP [38] at early times and the suppression of the spectrum as a whole at late times.

To be more specific, consider increasing the time from $\tau=0$ up to the maximal value $\tau_{L}=\sqrt{2 x_{c}}$, where we recall that $x_{c}>1$. When $\tau \rightarrow 0$, the r.h.s. of eq. (2.8) approaches $\delta(x-1)$, as it should. As long as $\tau$ is small enough for $\pi \bar{\alpha}^{2} \tau^{2} \ll 1$, the spectrum exhibits a pronounced peak in the vicinity of $x=1$, which describes the leading particle: the maximum of this peak lies at $x_{p}$ with $1-x_{p} \simeq(2 \pi / 3) \bar{\alpha}^{2} \tau^{2}$ and its width $\Delta x$ around $x_{p}$ is of order $\pi \bar{\alpha}^{2} \tau^{2}$. The fact that the peak gets displaced below 1 is a consequence of the Gaussian factor in eq. (2.8), which strongly suppresses the spectrum for $x$ close to 1 , within a window

$$
1-x \lesssim \pi \bar{\alpha}^{2} \tau^{2} \ll 1
$$

The physical origin of this suppression is quite clear: for $x$ close to 1 , the quantity $\epsilon \equiv$ $(1-x) E$ is the energy lost by the LP via radiation. According to eq. (2.10), this has a typical value $\epsilon(t) \simeq 2 \pi \omega_{s}(t)$, where $\omega_{s}(t)=\bar{\alpha}^{2} \hat{q} t^{2} / 2$ is recognized as the non-perturbative scale for multiple branchings during a time $t$ (recall the discussion after eq. (2.7)). That is, the LP radiates relatively soft gluons with $\omega \sim \omega_{s}(t)$, for which the emission probability is of $\mathcal{O}(1)$, and thus loses an energy of order $\omega_{s}(t)$. This energy loss is further enhanced by a rather large numerical factor $2 \pi$, which can be interpreted as the average number of gluons with energy $\omega \sim \omega_{s}(t)$ that are emitted by the LP during a time interval $t$. This interpretation will be supported by other findings below.

Let us now increase $\tau$ towards larger values $\pi \bar{\alpha}^{2} \tau^{2} \gtrsim 1$. Then the Gaussian suppression extends to all values of $x$, the LP peak gets washed out - it broadens, it moves towards smaller values of $x$, and its height is decreasing - and eventually disappears from the spectrum. One can say that a LP with energy $E \lesssim \bar{\alpha}^{2} \omega_{c}$ has a finite 'lifetime' inside the medium, of order $\Delta \tau \sim 1 / \bar{\alpha}$ or, in physical units (cf. eq. (2.5)),

$$
\Delta t \sim \frac{1}{\bar{\alpha}} \sqrt{\frac{E}{\hat{q}}} .
$$

More precisely, this means that the LP has fragmented into gluons which carry a sizable fraction of its original energy $E$. Via successive branchings, the energy gets degraded to lower and lower values of $x$, and it is interesting to understand this evolution in more detail. A priori, one might expect this energy to accumulate in the small- $x$ part of the spectrum, and notably at $x \lesssim x_{s}(\tau) \equiv \bar{\alpha}^{2} \tau^{2}$ (corresponding to $\omega \lesssim \omega_{s}(t)$ ), but eq. (2.8) shows that this is actually not the case: for $x \ll 1$, eq. (2.8) reduces to

$$
D(x, \tau) \simeq \frac{\bar{\alpha} \tau}{\sqrt{x}} \mathrm{e}^{-\pi \bar{\alpha}^{2} \tau^{2}}
$$




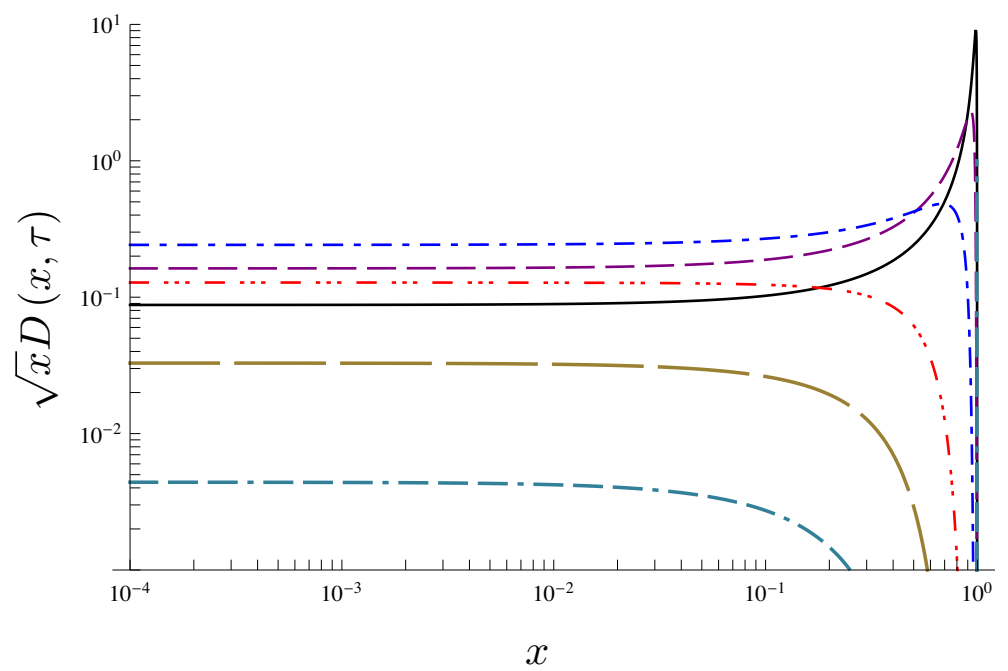

Figure 2. Plot (in log-log scale) of $\sqrt{x} D(x, \tau)$, with $D(x, \tau)$ given by eq. (2.8), as a function of $x$ for various values of $\tau$ : solid (black): $\tau=0.3$; dashed (purple): $\tau=0.6$; dashed-dotted (blue): $\tau=1.3$; dashed-triple dotted (red): $\tau=2.5$; long-dashed (brown): $\tau=3.5$; triple dashed-dotted (green): $\tau=4.5$. We use $\bar{\alpha}=0.3$.

which has exactly the same shape in $x$ as the small- $x$ limit of the BDMPSZ spectrum, eq. (2.9). In fact, eq. (2.12) formally looks like the BDMPSZ spectrum produced via a single emission by the LP, times a Gaussian factor describing the decay of the LP with increasing time. This interpretation seems to imply that multiple branchings are not important at small $x$, but from the discussion following eq. (2.7) we know that this cannot be true: after a time $t$, the single-branching probability becomes of order one for all the soft modes obeying $x<x_{s}(\tau)$. This last condition can also be inferred from eq. (2.9): when $x \sim x_{s}(\tau) \ll 1$, the BDMPSZ spectrum becomes of $\mathcal{O}(1)$.

We are thus facing an apparent paradox - in spite of the importance of multiple branching, the energy does not get accumulated in the bins of the spectrum at small $x-$ which finds its solution in the phenomenon of wave turbulence [16]. The BDMPSZ spectrum at small $x$ is not modified by the fragmentation because this represents a fixed point of the rate equation $(2.4)$ at small $x \ll 1$ : the branching term $\mathcal{I}[D]$ vanishes (meaning that the 'gain' and 'loss' terms compensate each other) when evaluated with the 'scaling' spectrum $D_{\text {sc }}(x) \equiv 1 / \sqrt{x}$. This represents the Kolmogorov-Zakharov (KZ) spectrum [30, 31] for the branching process at hand. In turn, the existence of this fixed point implies that, via successive branchings, the energy gets transmitted from large $x$ to small $x$, without accumulating at any intermediate value of $x$ : it rather accumulates into a condensate at $x=0$.

This is illustrated in figure 2, where the exact solution (2.8) is represented as a function of $x$ for several values of $\tau$, up to relatively large values, such that $\pi \bar{\alpha}^{2} \tau^{2} \gtrsim 1$. The LP peak is well pronounced at early times $\tau \lesssim 1$, but it is totally washed out at later times $\tau \gtrsim 1 / \bar{\alpha}$, where the spectrum is seen to be suppressed as a whole.

The energy flow can also be studied analytically, on the basis of eq. (2.8). To that aim, we consider the energy balance between spectrum and flow. The energy fraction contained 
in the spectrum after a time $\tau$ is computed as [16]

$$
\mathcal{E}(\tau)=\int_{0}^{1} \mathrm{~d} x D(x, \tau)=\mathrm{e}^{-\pi \bar{\alpha}^{2} \tau^{2}}
$$

and decreases with time. The difference

$$
\mathcal{E}_{\text {flow }}(\tau) \equiv 1-\mathcal{E}(\tau)=1-\mathrm{e}^{-\pi \bar{\alpha}^{2} \tau^{2}}
$$

is the energy fraction carried by the flow, i.e. by the multiple branchings, and which formally ends up in a condensate at $x=0$. For sufficiently large times $\bar{\alpha} \tau \gtrsim 1$, this can be as large as the total initial energy of the LP. It is also interesting to consider the small time limit of eq. (2.14), that is

$$
\mathcal{E}_{\text {flow }}(\tau) \simeq \pi \bar{\alpha}^{2} \tau^{2}=2 \pi x_{s}(\tau) \text { for } \pi \bar{\alpha}^{2} \tau^{2} \ll 1 .
$$

This formula admits a simple physical interpretation: $v_{0} \equiv 2 \pi$ is the average number of primary gluons with energies of the order of $\omega_{s}(t)=\bar{\alpha}^{2} \hat{q} t^{2} / 2$ that are emitted by the leading particle during a time $t$. Remarkably, this number is independent of both $t$ or $\bar{\alpha}$. This is possible since such soft gluon emissions occur with probability of order one during an interval of order $t$. Stated differently, the typical time interval between two successive emissions with energies of order $\omega_{s}(t)$ is itself of order $t$. This interval $\Delta t$ can be estimated from the condition that $\Delta \mathcal{P}_{\text {br }} \sim \mathcal{O}(1)$, with $\Delta \mathcal{P}_{\text {br }}$ given by eq. (2.7); this gives $\Delta t(\omega) \sim(1 / \bar{\alpha}) t_{\mathrm{br}}(\omega)$ and therefore $\Delta t \sim t$ when $\omega \sim \omega_{s}(t)$, as anticipated. After being emitted, the primary gluons with $\omega \sim \omega_{s}(t)$ rapidly cascade into even softer gluons and eventually transmit, after a time $\Delta t \sim t$, their whole energy to the very soft quanta which compose the flow.

\subsection{Energy flux, turbulence, and thermalization}

The physical interpretation of eq. (2.14) in terms of multiple branchings and, in particular, its relation to turbulent flow become more transparent if one studies a more differential quantity, the energy flux $\mathcal{F}\left(x_{0}, \tau\right)$. This is defined as the rate for energy transfer from the region $x>x_{0}$ to the region $x<x_{0}$. Since the energy in the region $x>x_{0}$ is decreasing with time, via branchings, it is natural to define the flux as the following, positive, quantity

$$
\mathcal{F}\left(x_{0}, \tau\right) \equiv-\frac{\partial \mathcal{E}^{>}\left(x_{0}, \tau\right)}{\partial \tau}=\frac{\partial \mathcal{E}^{<}\left(x_{0}, \tau\right)}{\partial \tau},
$$

where $\mathcal{E}^{>}\left(x_{0}, \tau\right)$ is the energy fraction contained in the bins of the spectrum with $x>x_{0}$, that is,

$$
\mathcal{E}^{>}\left(x_{0}, \tau\right)=\int_{x_{0}}^{1} \mathrm{~d} x D(x, \tau),
$$

whereas the complementary quantity $\mathcal{E}<\left(x_{0}, \tau\right)$ is the energy fraction carried by the modes with $x<x_{0}$. In turn, $\mathcal{E}^{<}\left(x_{0}, \tau\right)$ is the sum of two contributions: the flow energy (2.14) and the energy contained in the bins of the spectrum at $x<x_{0}$; that is,

$$
\mathcal{E}^{<}\left(x_{0}, \tau\right)=1-\mathcal{E}^{>}\left(x_{0}, \tau\right)=\mathcal{E}_{\text {flow }}(\tau)+\int_{0}^{x_{0}} \mathrm{~d} x D(x, \tau) .
$$




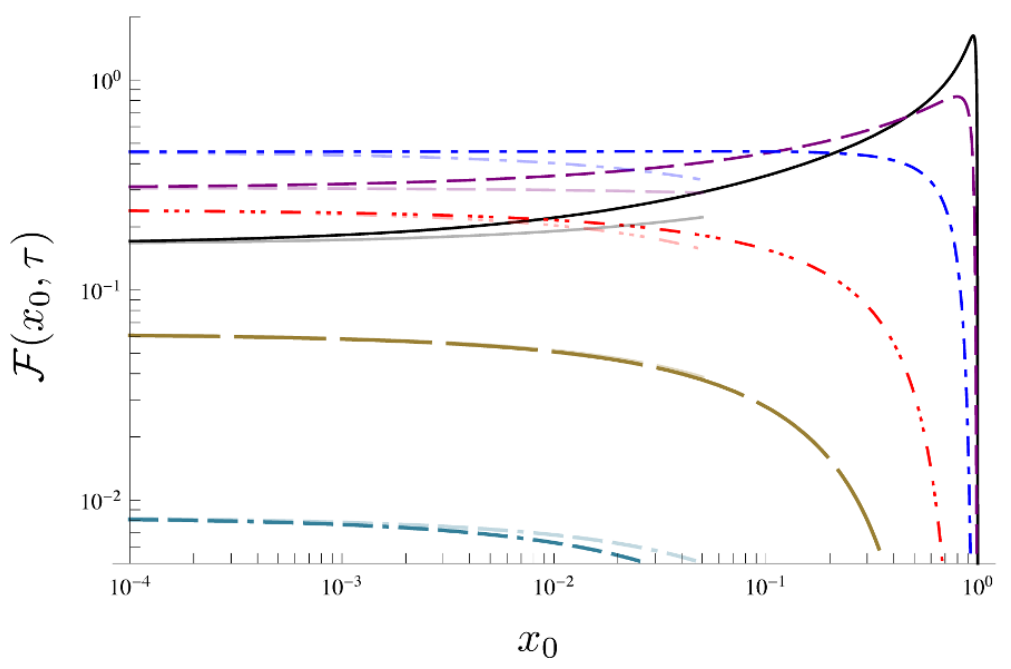

Figure 3. Plot (in log-log scale) of the energy flux $\mathcal{F}\left(x_{0}, \tau\right)$, cf. eq. (2.16), as a function of $x_{0}$ for various values of $\tau$. We use the same conventions as in figure 2. The thin curves, which are drawn for $x_{0} \leq 0.05$, represent the approximation in eq. (2.19), which is valid at small $x_{0}$.

Using the above definitions together with eq. (2.8) for $D(x, \tau)$, it is straightforward to numerically compute the energy flux $\mathcal{F}\left(x_{0}, \tau\right)$, with the results displayed in figure 3 . For a physical discussion, it is convenient to focus on the behavior at small $x_{0} \ll 1$. In that region, one can use eq. (2.18) together with the small- $x$ approximation to the spectrum, eq. (2.12), to deduce the analytic estimate

$$
\mathcal{F}\left(x_{0}, \tau\right) \simeq\left[2 \pi \bar{\alpha}^{2} \tau+2 \bar{\alpha} \sqrt{x_{0}}\left(1-2 \pi \bar{\alpha}^{2} \tau^{2}\right)\right] \mathrm{e}^{-\pi \bar{\alpha}^{2} \tau^{2}} .
$$

The first term within the square brackets, which is independent of $x_{0}$, is the flow contribution,

$$
\mathcal{F}_{\text {flow }}(\tau) \equiv \frac{\partial \mathcal{E}_{\text {flow }}(\tau)}{\partial \tau}=2 \pi \bar{\alpha}^{2} \tau \mathrm{e}^{-\pi \bar{\alpha}^{2} \tau^{2}}
$$

while the second term, proportional to $\sqrt{x_{0}}$, is the rate at which the energy changes in the region of the spectrum at $x \leq x_{0}$. Clearly, the flow component in eq. (2.20) dominates over the non-flow one at sufficiently small values of $x_{0}$, such that $x_{0} \lesssim x_{s}(\tau)=\bar{\alpha}^{2} \tau^{2}$. This is also visible in figure 3 , where the various curves become indeed flat at sufficiently small $x_{0}$. The function $\mathcal{F}_{\text {flow }}(\tau)$ is represented in figure 6 (see the brown, long-dashed, curve in that figure). It exhibits a rather wide maximum at a value $\tau \sim 1 / \bar{\alpha}$, which corresponds to the average lifetime of the LP before undergoing a democratic branching (cf. eq. (2.11)).

A uniform energy flux (in the sense of being independent of $x$, or of $\omega$ ) is the distinguished signature of (wave) turbulence [30,31]. It physically means that the energy flows through the spectrum without accumulating at intermediate values of $x$. It is intuitively clear that a quasi-uniform flux requires the branchings to be quasi-local in $x$ (or 'quasidemocratic'). Since, if the typical branchings were strongly asymmetric, then after each branching most of the energy would remain in the parent gluon and the energy would accumulate in the bins at large $x$. It is also quite clear, in view of the splitting law (2.2), that 
in the non-perturbative region at $x \lesssim x_{s}(\tau)$ the branchings are indeed quasi-democratic: a gluon with energy $x \sim x_{s}(\tau)$ splits with probability of $\mathcal{O}(1)$ during a time interval $\tau$ irrespective of the value $z$ of the splitting fraction. Hence, there is no reason why special values like $z \ll 1$ or $1-z \ll 1$ should be favored. A more elaborate argument for democratic branchings will be presented in section 3.4.

The locality of the interactions is a fundamental property of turbulence [30, 31]. In the traditional turbulence problem, where the energy is injected by a time-independent source which is localized in energy and produces a steady spectrum, this property ensures that the energy spectrum in the 'inertial range' (i.e. sufficiently far away from the source) can be expressed in terms of the (steady) flux $\mathcal{F}$ and a special power-like spectrum, the 'Kolmogorov-Zakharov spectrum', which is a fixed-point of the 'collision term'. In the case of hydrodynamic turbulence in $3+1$ dimensions, this relation between the energy spectrum and the flux is known as the 'Kolmogorov-Obukhov spectrum'.

For the problem at hand, where the 'source' is the leading particle originally localized at $x=1$, the 'inertial region' corresponds to $x \ll 1$, the 'collision term' term is the branching term $\bar{\alpha} \mathcal{I}[D]$, and the fixed-point solution is the scaling spectrum $D_{\mathrm{sc}}(x)=1 / \sqrt{x}$. But unlike for the more conventional set-up, our current problem is clearly not stationary: the 'source' (the LP) loses energy and can even disappear at large times, so both the spectrum and the energy flux have non-trivial time dependencies. Notwithstanding, it turns out that the fundamental relation alluded to above, between the energy spectrum and the flux, also holds for the time-dependent physical problem at hand. Namely, by inspection of eqs. (2.12) and (2.20), it is clear than one can write

$$
D(x, \tau) \simeq \frac{1}{2 \pi \bar{\alpha}} \frac{\mathcal{F}_{\text {flow }}(\tau)}{\sqrt{x}} \quad \text { for } \quad x \ll 1 .
$$

This is a version of the Kolmogorov-Obukhov relation adapted to the current problem and generalized to a time-dependent situation. Note that eq. (2.21) involves only the flow contribution to the flux, albeit this relation holds for any $x \ll 1$ and not only in the 'non-perturbative' sector at $x \lesssim x_{s}(\tau)$. At this level, the relation (2.21) might look fortuitous, but in section 3.4 we shall present a general argument showing that it has a deep physical motivation.

So far, we have implicitly assumed that the branching dynamics as described by eq. (2.4) extends all the way down to $x=0$, that is, it includes arbitrarily soft gluons. In reality, the dynamics should change at sufficiently low energies, for various reasons. First, when the gluons in the cascade become as soft as the medium constituents - that is, their energies become comparable to the temperature $T$ - they rapidly thermalize via collisions in the medium and thus 'disappear' from the cascade. Second, the BDMPSZ branching law (2.2) assumes the dominance of multiple soft scattering and hence it ceases to be valid when the branching time $t_{\mathrm{br}}(z, \omega)$ becomes as low as the mean free path $\ell$ between successive collisions in the medium. This condition restricts the gluon energies to values $\omega \gtrsim \omega_{\mathrm{BH}} \equiv \hat{q} \ell^{2} / 2$. For a weakly coupled quark-gluon plasma, the 'Beithe-Heitler' scale $\omega_{\mathrm{BH}}$ is comparable to the temperature $T$. (Indeed, one has $\hat{q} \sim \bar{\alpha}^{2} T^{3}$ and $\ell \sim(\bar{\alpha} T)^{-1}$ to parametric accuracy.) With this example in mind, we shall not distinguish between these 
two scales anymore, but simply assume that the dynamics described by eq. (2.4) applies for all the energies $\omega \gtrsim T$, i.e., for all $x \gtrsim x_{\text {th }} \equiv T / E$. In all the interesting problems, the thermal scale $x_{\text {th }}$ is small enough to allow for multiple branchings: $x_{\text {th }} \ll x_{s}=\bar{\alpha}^{2} x_{c}$. For instance, in the case of a weakly coupled plasma, the above condition is tantamount to $L \gg \ell / \bar{\alpha} \sim\left(\bar{\alpha}^{2} T\right)^{-1}$, which is indeed satisfied since the interesting values for $L$ are much larger than the typical relaxation time $\lambda_{\text {rel }} \sim\left(\bar{\alpha}^{2} T\right)^{-1}$ of the plasma.

So far, we have implicitly assumed that the thermalization mechanism acts as a 'perfect sink' at $x \sim x_{\mathrm{th}}$. (A similar assumption was made in the 'bottom-up' scenario for thermalization [22] and in the Monte-Carlo implementation of the rate equation in the event generator MARTINI [24].) That is, the surrounding medium absorbs the energy from the cascade at a rate equal to the relevant flux $\mathcal{F}\left(x_{\mathrm{th}}, \tau\right)$, without modifying the branching dynamics at higher values $x \gg x_{\mathrm{th}}$. This is a rather standard assumption in the context of turbulence and is also well motivated for the problem at hand, as we now explain. To that aim, one should compare the relaxation time $\lambda_{\text {rel }} \sim\left(\bar{\alpha}^{2} T\right)^{-1}$ aforementioned, which represents the characteristic thermalization time at weak coupling, with the lifetime $\Delta t(\omega)$ of a gluon generation (the time interval between two successive branchings) for gluons with energy $\omega \sim T$. This $\Delta t(\omega)$ can be estimated as explained at the end of section 2.2, and reads, parametrically,

$$
\Delta t(\omega) \sim \frac{1}{\bar{\alpha}} t_{\mathrm{br}}(\omega) \sim \frac{1}{\bar{\alpha}} \sqrt{\frac{\omega}{\hat{q}}} .
$$

Using $\omega \sim T$ and the perturbative estimate $\hat{q} \sim \bar{\alpha}^{2} T^{3}$, one deduces $\Delta t \sim\left(\bar{\alpha}^{2} T\right)^{-1} \sim \lambda_{\text {rel }}$. We thus conclude that the physics of thermalization is as efficient in dissipating the energy as the turbulent flow. This implies that there should be no energy pile-up towards the low-energy end of the cascade.

Under these assumptions, it is interesting to compute the total energy lost by the cascade towards the medium, i.e. 'the energy which thermalizes'. This is the same as the energy which has the crossed the bin $x_{\text {th }}$ during the overall time $\tau_{L}$, namely (cf. eq. (2.18))

$$
\mathcal{E}_{\text {th }} \equiv \mathcal{E}^{<}\left(x_{\text {th }}, \tau_{L}\right) \simeq 1-\mathrm{e}^{-\pi \bar{\alpha}^{2} \tau_{L}^{2}}+2 \bar{\alpha} \tau_{L} \sqrt{x_{\mathrm{th}}} \mathrm{e}^{-\pi \bar{\alpha}^{2} \tau_{L}^{2}},
$$

where the approximate equality holds since $x_{\text {th }} \ll 1$. eq. (2.23) is recognized as the sum of the flow energy, eq. (2.14), and of the energy that would be contained in the spectrum at $x \leq x_{\text {th }}$, cf. eq. (2.12). Using $\tau_{L}=\sqrt{2 x_{c}}$ and $x_{\text {th }} \ll x_{s}=\bar{\alpha}^{2} x_{c}$, it is easy to check that the flow component dominates over the spectrum piece, and hence $\mathcal{E}_{\text {th }} \simeq \mathcal{E}_{\text {flow }}\left(\tau_{L}\right)$. This implies that the energy lost by the gluon cascade towards the medium is independent of the details of the thermalization process, like the precise value of $x_{\mathrm{th}}$. This universality too is a well known feature of a turbulent process [30, 31].

\section{The high-energy regime}

With this section, we begin the study of the main physical problem of interest for us in this paper, namely the gluon cascade produced in the medium by a very energetic leading particle, with original energy $E \gg \omega_{c}$. The main new ingredient as compared to the previous discussion is a kinematical restriction on the primary gluon emissions that can 
be triggered by interactions in the medium: the energy $\omega$ of the gluons emitted by the LP cannot exceed a value $\omega_{c}$ in order for the respective formation time $t_{\mathrm{br}}(\omega)$ to remain smaller than $L$. When $x_{c} \equiv \omega_{c} / E \ll 1$, this restriction has important consequences: it implies that the LP loses only a small fraction of its total energy, of order $\bar{\alpha} x_{c} \ll 1$. Our main focus in what follows will not be on this average energy lost by the LP (this is well understood within the original BDMPSZ formalism, including multiple soft emissions of primary gluons [38]), but rather on the further evolution of this radiation via multiple branchings and the associated flow of energy towards small values of $x$ and large angles.

\subsection{The coupled rate equations}

Since the radiation is restricted to relatively low energies $\omega \leq \omega_{c} \ll E$, or $x \leq x_{c} \ll 1$, it is clear that the part of the spectrum at higher energies $x_{c}<x<1$ has to be associated with the LP. This makes it natural to decompose the overall spectrum as

$$
D(x, \tau)=\left[\Theta\left(x-x_{c}\right)+\Theta\left(x_{c}-x\right)\right] D(x, \tau) \equiv D_{\mathrm{LP}}(x, \tau)+D_{\text {rad }}(x, \tau) .
$$

In reality, the LP piece $D_{\mathrm{LP}}(x, \tau)$ is a rather narrow peak located in the vicinity of $x=1$ (see below), so there is a large gap between the two components of the spectrum.

The evolution of the radiation via successive branchings involves no special constraint, so the respective rate equation can be obtained simply by replacing $D(x, \tau)$ according to eq. (3.1) in the r.h.s. of the general equation eq. (2.4) (restricted to $x<x_{c}$, of course). This yields

$$
\frac{\partial D_{\text {rad }}(x, \tau)}{\partial \tau}=\mathcal{S}(x, \tau)+\bar{\alpha} \int \mathrm{d} z \mathcal{K}(z)\left\{\sqrt{\frac{z}{x}} D_{\operatorname{rad}}\left(\frac{x}{z}, \tau\right)-\frac{z}{\sqrt{x}} D_{\operatorname{rad}}(x, \tau)\right\},
$$

where the source $\mathcal{S}(x, \tau)$ is the energy per unit time and per unit $x$ radiated by the LP:

$$
\mathcal{S}(x, \tau) \equiv \bar{\alpha} \int \mathrm{d} z \mathcal{K}(z) \sqrt{\frac{z}{x}} D_{\mathrm{LP}}\left(\frac{x}{z}, \tau\right) .
$$

It is here implicitly understood that this source has support at $x \leq x_{c}$ and that it acts over a limited interval in time, at $0 \leq \tau \leq \tau_{L} \equiv \sqrt{2 x_{c}}$, which is moreover small, $\tau_{L} \ll 1$, in the high-energy regime of interest. The integral over $z$ in the gain term of eq. (3.2) is restricted to $x / x_{c}<z<1$, where the lower limit is introduced by the support of the function $D_{\text {rad }}(x / z, \tau)$.

In the rate equation for the leading particle, one needs to enforce the condition that the radiated gluons have energy fractions smaller than $x_{c}$. The ensuing equation reads (with $x>x_{c}$ )

$$
\begin{aligned}
\frac{\partial D_{\mathrm{LP}}(x, \tau)}{\partial \tau}=\bar{\alpha} \int \mathrm{d} z \mathcal{K}(z)\left\{\Theta\left(z-\frac{x}{x+x_{c}}\right) \sqrt{\frac{z}{x}} D_{\mathrm{LP}}\left(\frac{x}{z}, \tau\right)\right. \\
\left.-\frac{z}{\sqrt{x}} D_{\mathrm{LP}}(x, \tau)\left[\Theta\left(z-1+\frac{x_{c}}{x}\right)+\Theta\left(\frac{x_{c}}{x}-z\right)\right]\right\}
\end{aligned}
$$

where the various $\Theta$-functions enforce the kinematical constraint: in the gain term, one requires that the unmeasured gluon emitted (with splitting fraction $1-z$ ) by the LP (with 
initial energy fraction $x / z)$ be softer than $x_{c}$ : $(1-z)(x / z)<x_{c} \Longrightarrow z>x /\left(x+x_{c}\right)$. In the loss term, one requires that one of the daughter gluons be soft: either $z x<x_{c}$, or $(1-z) x<x_{c}$.

As it should be clear from the previous discussion, the functions $D_{\mathrm{LP}}(x, \tau)$ and $D_{\text {rad }}(x, \tau)$ at any time $\tau<\tau_{L}$ also depend upon $x_{c}$, hence upon the overall size $L$ of the medium, via the kinematical constraints on the gluon emissions. This shows that the dynamics in this high energy regime is non-local in time; e.g., the branching rate in eq. (3.4) 'knows' about the maximal time $\tau_{L}$ via the various $\Theta$-functions, which involve $x_{c}$. This property reflects a true non-locality of the underlying quantum dynamics: it takes some time to emit a gluon and this time cannot be larger than $L$. Accordingly, at any $\tau<\tau_{L}$, one should only initiate emissions whose energies are smaller than $\omega_{c}$ : gluon fluctuations with higher energies would have no time to become on-shell. The kinematical constraint $\omega \leq \omega_{c}$ reflects only in a crude way the actual non-locality of the quantum emissions. The classical description at hand, as based on rate equations, is truly appropriate only for the sufficiently soft emissions with small formation times $t_{\mathrm{br}}(\omega) \ll L$. Fortunately, these are the most important emissions for the physics problems that we shall here address.

In the zeroth order approximation, which is strictly valid as $\tau \rightarrow 0$, one can use $D_{\mathrm{LP}}(x, \tau)=\delta(1-x)$, and then the source in eq. (3.3) reduces to the BDMPSZ spectrum, as expected:

$$
\mathcal{S}_{0}(x) \equiv \bar{\alpha} x \mathcal{K}(x) \simeq \frac{\bar{\alpha}}{\sqrt{x}}
$$

In writing the second, approximate, equality we have used the fact that $x$ is small, $x \leq$ $x_{c} \ll 1$, to simplify the expression of the splitting kernel (cf. eq. (2.6)): $\mathcal{K}(x) \simeq x^{-3 / 2}$ for $x \ll 1$.

We shall now argue that the expression (3.5), which is time-independent, remains a good approximation for all the times $\tau$ of interest. Of course, the spectrum $D_{\mathrm{LP}}(x, \tau)$ of the LP changes quite fast with increasing $\tau$, notably due to the prompt radiation of very soft quanta with energy fractions $x \lesssim x_{s}(\tau)=\bar{\alpha}^{2} \tau^{2}$. This leads to a broadening of the LP peak on the scale $\Delta x \sim \bar{\alpha}^{2} \tau^{2} \lesssim \bar{\alpha}^{2} x_{c} \ll 1$, similar to that exhibited by eq. (2.8) at small times. Yet, the probability to emit a relatively hard gluon with $x \sim x_{c}$ is very small, of $\mathcal{O}(\bar{\alpha})$. Accordingly, the support of the function $D_{\mathrm{LP}}(x, \tau)$ remains limited to a narrow band at $1-x_{c} \lesssim x<1$, which is well separated from the radiation spectrum at $x<x_{c}$. Hence, the integration over $z$ in eq. (3.3) is effectively restricted to a narrow range close to $x$, namely $x<z<x /\left(1-x_{c}\right)$, and the integral can be approximated as

$$
\mathcal{S}(x, \tau) \simeq \bar{\alpha} x \mathcal{K}(x) \int \mathrm{d} x^{\prime} D_{\mathrm{LP}}\left(x^{\prime}, \tau\right) \simeq \frac{\bar{\alpha}}{\sqrt{x}}\left[1+\mathcal{O}\left(x, \bar{\alpha} x_{c}\right)\right] .
$$

Here we have used the fact that the overall strength of the function $D_{\mathrm{LP}}(x, \tau)$, i.e. the energy fraction carried by the LP after a time $\tau$, can be estimated as

$$
\mathcal{E}_{\mathrm{LP}}(\tau) \equiv \int \mathrm{d} x D_{\mathrm{LP}}(x, \tau) \simeq 1-2 \bar{\alpha} \tau \sqrt{x_{c}},
$$

that is, the initial energy minus the energy lost via radiation (the integral of eq. (3.5) up to $x=x_{c}$ ). 


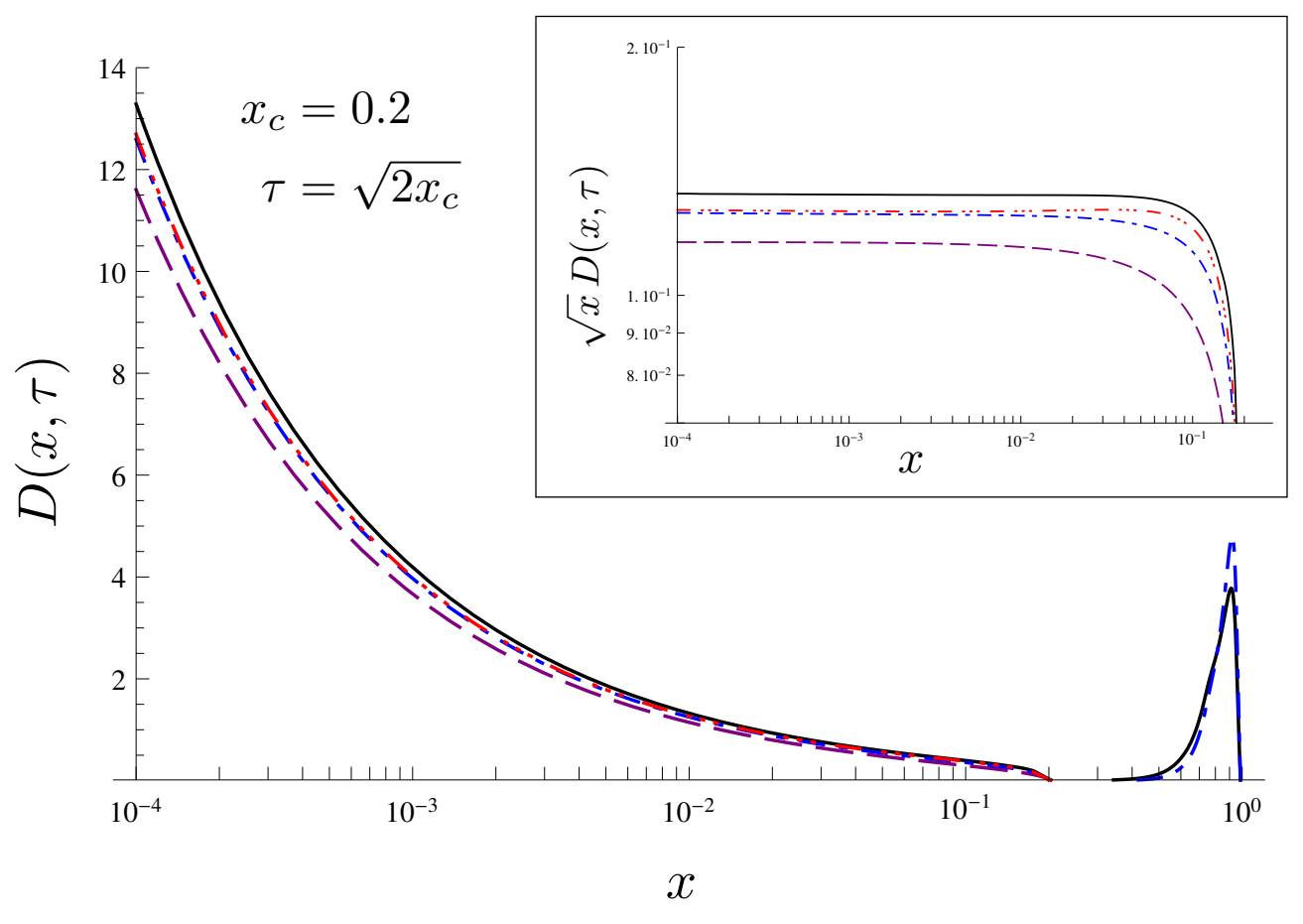

Figure 4. The full spectrum $D(x, \tau)=D_{\mathrm{LP}}(x, \tau)+D_{\mathrm{rad}}(x, \tau)$ obtained by numerically solving the coupled equations (3.2) and (3.4), versus the radiation spectrum predicted by eq. (3.8) with a source. We use both versions of the kernel, $\mathcal{K}$ and $\mathcal{K}_{0}$, together with $x_{c}=0.2$ and $\tau=\sqrt{2 x_{c}} \simeq 0.63$. (i) Simplified kernel $\mathcal{K}_{0}$ : black curve: eqs. (3.2)-(3.4); purple, dashed: eq. (3.8). (ii) Full kernel $\mathcal{K}$ : blue, dashed-dotted: eqs. (3.2)-(3.4); red, dashed-triple dotted: eq. (3.8). In the insert: the same plots (for the radiation part only) in log-log scale.

To summarize, after an evolution time $\tau_{L}$, the energetic LP loses only a small fraction $\bar{\alpha} \sqrt{x_{c}} \tau_{L} \sim \bar{\alpha} x_{c} \ll 1$ of its total energy and its spectral density remains peaked near $x=1$. Accordingly, it can be effectively treated as a steady source $\mathcal{S}_{0}(x)$ for the soft radiation at $x \ll 1$. This is verified in the plots in figure 4 , where we perform two types of comparisons: (i) between the evolution with the exact kernel $\mathcal{K}$ in eq. (2.6) and that with the simplified kernel $\mathcal{K}_{0}$, and (ii) between the solution to the coupled system of equations (3.2) and (3.4) and that to the effective equation with a source, i.e. eq. (3.2) with $\mathcal{S}(x, \tau) \rightarrow \mathcal{S}_{0}(x)$. As one can see in this plot, the two choices for the kernel lead indeed to results which are qualitatively similar and numerically very close to each other. Furthermore, the radiation spectrum at $x \leq x_{c}$ produced by the 'model' equation with a source is indeed close to the respective prediction of the coupled rate equations. (In fact, for the exact kernel $\mathcal{K}$, this similarity looks even more striking - the respective curves almost overlap with each other at sufficiently small $x$ - but in our opinion this is merely a coincidence.) In the next subsection, we shall construct an exact analytic solution for the equation with the source, for the case of the simplified kernel $\mathcal{K}_{0}$. 


\subsection{The radiation spectrum}

In the remaining part of this section, we shall concentrate on the solution to the following equation

$$
\begin{aligned}
\frac{\partial D_{\mathrm{rad}}(x, \tau)}{\partial \tau} & =\frac{\bar{\alpha}}{\sqrt{x}}+\bar{\alpha} \int \mathrm{d} z \mathcal{K}(z)\left\{\sqrt{\frac{z}{x}} D_{\text {rad }}\left(\frac{x}{z}, \tau\right)-\frac{z}{\sqrt{x}} D_{\text {rad }}(x, \tau)\right\} \\
& \equiv \mathcal{S}_{0}(x)+\bar{\alpha} \mathcal{I}\left[D_{\mathrm{rad}}\right](x, \tau)
\end{aligned}
$$

which, as above argued, offers a good approximation for the dynamics of the mediuminduced radiation by a leading particle with high energy $E \gg \omega_{c}$. This is an inhomogeneous equation with vanishing initial condition and can be solved with the help of the respective Green's function:

$$
D_{\text {rad }}(x, \tau)=\int_{x}^{x_{c}} \mathrm{~d} x_{1} \int_{0}^{\tau} \mathrm{d} \tau_{1} G\left(x, x_{1}, \tau-\tau_{1}\right) \mathcal{S}_{0}\left(x_{1}\right) .
$$

The Green's function $G\left(x, x_{1}, \tau\right)$ obeys the homogeneous version of eq. (3.8) with initial condition $G\left(x, x_{1}, \tau\right)=\delta\left(x-x_{1}\right)$.

From now on, we shall again restrict ourselves to the case of the simplified splitting kernel $\mathcal{K}_{0}(z)$, which we recall is obtained by replacing $f(z) \rightarrow 1$ in eq. (2.6). For this case, the Green's function $G\left(x, x_{1}, \tau\right)$ can be exactly computed, since it is closely related to the function $D(x, \tau)$ in eq. (3.10): both functions obey eq. (2.4), but with slightly different initial conditions. It is easy to check that the corresponding solutions are related via an appropriate rescaling of the variables:

$$
G\left(x, x_{1}, \tau\right)=\frac{1}{x_{1}} D\left(\frac{x}{x_{1}}, \frac{\tau}{\sqrt{x_{1}}}\right)=\sqrt{\frac{x_{1}}{x}} \frac{\bar{\alpha} \tau}{\left(x_{1}-x\right)^{3 / 2}} \exp \left\{-\frac{\pi \bar{\alpha}^{2} \tau^{2}}{x_{1}-x}\right\} .
$$

Since the source $\mathcal{S}_{0}\left(x_{1}\right)$ in eq. (3.9) is independent of time, the integral over $\tau_{1}$ involves only the Green's function and can be readily computed:

$$
\int_{0}^{\tau} \mathrm{d} \tau_{1} G\left(x, x_{1}, \tau-\tau_{1}\right)=\frac{1}{2 \pi \bar{\alpha}} \sqrt{\frac{x_{1}}{x\left(x_{1}-x\right)}}\left[1-\exp \left\{-\frac{\pi \bar{\alpha}^{2} \tau^{2}}{x_{1}-x}\right\}\right] .
$$

To also compute the integral over $x_{1}$, it is convenient to change the integration variable according to $u \equiv \pi \bar{\alpha}^{2} \tau^{2} /\left(x_{1}-x\right)$. One thus easily finds

$$
D_{\operatorname{rad}}(x, \tau)=\frac{\bar{\alpha} \tau}{\sqrt{x}} \frac{1}{2 \sqrt{\pi}} \int_{\zeta}^{\infty} \frac{\mathrm{d} u}{u^{3 / 2}}\left[1-\mathrm{e}^{-u}\right]=\frac{\bar{\alpha} \tau}{\sqrt{x}}\left\{\frac{1}{\sqrt{\pi}} \Gamma\left(\frac{1}{2}, \zeta\right)+\frac{1-\mathrm{e}^{-\zeta}}{\sqrt{\pi \zeta}}\right\}
$$

where

$$
\zeta \equiv \zeta\left(x_{c}-x, \tau\right) \equiv \frac{\pi \bar{\alpha}^{2} \tau^{2}}{x_{c}-x}
$$

and

$$
\Gamma\left(\frac{1}{2}, \zeta\right) \equiv \int_{\zeta}^{\infty} \frac{\mathrm{d} z}{\sqrt{z}} \mathrm{e}^{-z}=\sqrt{\pi}-\int_{0}^{\zeta} \frac{\mathrm{d} z}{\sqrt{z}} \mathrm{e}^{-z}=\sqrt{\pi}-\gamma\left(\frac{1}{2}, \zeta\right)
$$




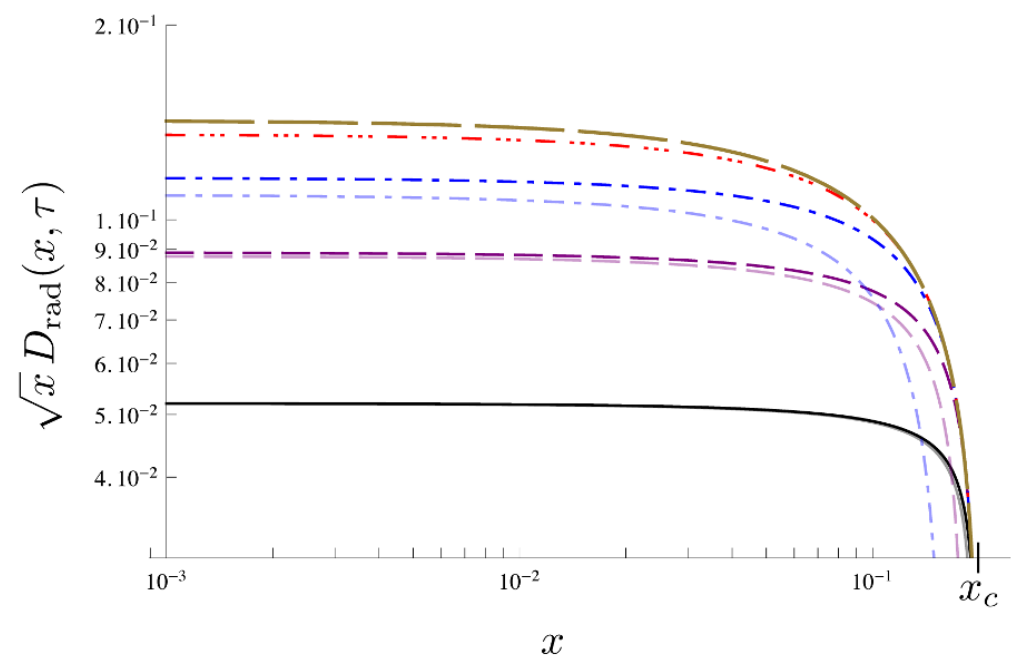

Figure 5. Plot (in log-log scale) of $D_{\operatorname{rad}}(x, \tau)$, cf. eq. (3.12), as a function of $x$ for $x_{c}=0.2$ and various values of $\tau$. The thick curves show the function $\sqrt{x} D_{\operatorname{rad}}(x, \tau)$ for $\tau=0.2$ (black, solid), $\tau=0.4$ (purple, dashed), $\tau=0.63$ (blue, dashed-dotted), and $\tau=1.0$ (red, dashed-triple dotted). Note that the maximal value for $\tau$ which is physically allowed is $\tau_{L}=\sqrt{0.4} \simeq 0.63$. The thin curves, shown for $\tau \leq \tau_{L}$, represent the corresponding approximations at small $\zeta\left(x_{c}-x, \tau\right)$, as obtained by keeping only the first 2 terms in the Taylor expansion in eq. (3.20). The enveloping curve (brown, long-dashed) is the limiting curve at large $\zeta$, cf. eq. (3.21).

is the upper incomplete Gamma function (whereas $\gamma(1 / 2, \zeta)$ is the respective lower function). Note that $D_{\text {rad }}(x, \tau)$ is also a function of the limiting energy fraction $x_{c}$, but in our notations this dependence is left implicit. A similar observation applies to all formulæ that appear in this section.

For what follows, it is also useful to single out the piece of the spectrum that would be produced by the source term alone, in the absence of branchings. Specifically, using eq. (3.14), we can write

$$
\begin{aligned}
D_{\mathrm{rad}}(x, \tau) & =\frac{\bar{\alpha} \tau}{\sqrt{x}}-\delta D_{\mathrm{br}}(x, \tau), \\
\delta D_{\mathrm{br}}(x, \tau) & \equiv \frac{\bar{\alpha} \tau}{\sqrt{x}}\left\{\frac{1}{\sqrt{\pi}} \gamma\left(\frac{1}{2}, \zeta\right)-\frac{1-\mathrm{e}^{-\zeta}}{\sqrt{\pi \zeta}}\right\} \equiv \frac{\bar{\alpha} \tau}{\sqrt{x}} h(\zeta),
\end{aligned}
$$

where the quantity $\delta D_{\mathrm{br}}(x, \tau)$ is the change in the spectrum due to multiple branchings and it is positive semi-definite, as one can easily check - meaning that the effect of branchings is a depletion in the spectrum, at any $x \leq x_{c}$. This depletion reflects the flow of energy from one parton generation to the next one, via parton branching - a phenomenon to which we shall return in the next subsection. But before doing that, let us discuss the radiation spectrum (3.12) in more detail.

This spectrum is depicted in figure 5 as a function of $x$ for various values of $\tau$. The different limiting behaviors can be also understood in analytic terms. To that aim, it is useful to notice a few properties of the function $h(\zeta)$. This function is monotonously increasing and interpolates between $h=0$ at $\zeta=0$ and $h \rightarrow 1$ as $\zeta \rightarrow \infty$. Furthermore, 
the ratio $h(\zeta) / \sqrt{\zeta}$ is an analytic function of $\zeta$ with infinite radius of convergence and a rapidly converging Taylor expansion:

$$
\sqrt{\frac{\pi}{\zeta}} h(\zeta)=\int_{0}^{1} \mathrm{~d} u u^{-1 / 2} \mathrm{e}^{-\zeta u}-\frac{1-\mathrm{e}^{-\zeta}}{\zeta}=1-\frac{1}{6} \zeta+\frac{1}{30} \zeta^{2}+\mathcal{O}\left(\zeta^{3}\right) .
$$

Finally, for large $\zeta$, one finds the asymptotic behavior

$$
1-h(\zeta)=\frac{1}{\sqrt{\pi \zeta}}+\cdots
$$

where the dots stand for terms which are exponentially suppressed.

Returning to the spectrum in eq. (3.15), we first observe that at small $x \ll x_{c}$ this reduces to the scaling spectrum $D_{\mathrm{sc}}(x)=1 / \sqrt{x}$ — the expected fixed point of the branching dynamics at small $x$. Indeed, when $x \ll x_{c}$, one can approximate $\zeta\left(x_{c}-x, \tau\right) \simeq \zeta\left(x_{c}, \tau\right)$ and therefore

$$
D_{\mathrm{rad}}(x, \tau) \simeq \frac{\bar{\alpha} \tau}{\sqrt{x}}\left[1-h\left(\zeta_{0}\right)\right], \quad \zeta_{0} \equiv \zeta\left(x_{c}, \tau\right)=\frac{\pi \bar{\alpha}^{2} \tau^{2}}{x_{c}} .
$$

Interestingly, at the end of the evolution, i.e. for $\tau=\tau_{L}=\sqrt{2 x_{c}}$, eq. (3.18) reduces to the BDMPSZ spectrum times a function of the QCD coupling $\bar{\alpha}$, which is strictly smaller than 1 and which expresses the reduction in the spectrum due to multiple branchings:

$$
D_{\mathrm{rad}}\left(x, \tau_{L}\right) \simeq \bar{\alpha} \sqrt{\frac{2 x_{c}}{x}}\left[1-h\left(2 \pi \bar{\alpha}^{2}\right)\right] \text { for } \quad x \ll x_{c} .
$$

Consider now larger values of $x$, where the deviations from the scaling spectrum start to be important. As long as $x$ is not too close to $x_{c}$, such that $\zeta \lesssim 1$, the spectrum can be expanded in powers of $\zeta$, with the help of eq. (3.16). One thus finds

$$
D_{\operatorname{rad}}(x, \tau)=\frac{\bar{\alpha} \tau}{\sqrt{x}}\left\{1-\frac{\bar{\alpha} \tau}{\sqrt{x_{c}-x}}+\frac{\pi}{6}\left(\frac{\bar{\alpha} \tau}{\sqrt{x_{c}-x}}\right)^{3}+\cdots\right\} \quad \text { when } \quad \zeta\left(x_{c}-x, \tau\right) \lesssim 1,
$$

where the dots stand for terms of $\mathcal{O}\left(\zeta^{5 / 2}\right)$ and higher. This expansion is rapidly converging for any $\zeta \lesssim 1$. Given that $\zeta\left(x_{c}, \tau_{L}\right)=2 \pi \bar{\alpha}^{2}$ is a relatively small number $\left(2 \pi \bar{\alpha}^{2} \simeq 0.6\right.$ for $\bar{\alpha}=0.3$ ), we expect a limited expansion like eq. (3.20) to be quite accurate for any $\tau \lesssim \tau_{L}$ and for $x$ values in the bulk. And indeed, the curves obtained by keeping just the first 2 terms in this expansion provide an excellent approximation to the full curves in figure 5 for any $\tau \leq \tau_{L}$, except of course for $x$ very close to $x_{c}$. Notice that the inclusion of the first correction in eq. (3.20), which expresses the dominant effect of the multiple branchings at small $\bar{\alpha} \tau$, is truly essential in order to obtain such a good agreement. Indeed, for $\tau \sim \tau_{L}$ and $x$ values in the bulk, that correction is numerically important, of relative order $\bar{\alpha} \tau_{L} / \sqrt{x_{c}}=\sqrt{2} \bar{\alpha} \simeq 0.4$.

The expansion in eq. (3.20) breaks down when the first correction becomes of $\mathcal{O}(1)$ or larger, namely for $x_{c}-x \lesssim \bar{\alpha}^{2} \tau^{2}$. This is in agreement with the fact that the emission 
of very soft gluons, with energy fractions $x \lesssim x_{s}(\tau)=\bar{\alpha}^{2} \tau^{2}$, is non-perturbative. To investigate the effect of such emissions via analytic approximations, let us consider the behavior near the endpoint of the spectrum, at $x \rightarrow x_{c}$. In that limit, one has $\zeta \gg 1$, so one can use eq. (3.17) to deduce

$$
D_{\mathrm{rad}}(x, \tau) \simeq \frac{1}{\pi} \sqrt{\frac{x_{c}-x}{x}} \text { when } \quad \zeta\left(x_{c}-x, \tau\right) \gg 1 .
$$

This result is time-independent and shows that the spectrum vanishes when $x \rightarrow x_{c}$ at any time $\tau$. This demonstrates the efficiency of the soft branchings in depleting the spectrum near its endpoint. The energy which is transferred in this way towards the bins at $x<x_{c}$ cannot be compensated by a corresponding flow of energy coming from the bins at $x>x_{c}$, since the spectrum ends at $x_{c}$.

The steady spectrum in eq. (3.21) also represents the limiting curve for the function $D_{\text {rad }}(x, \tau)$ in the formal large-time limit at $\zeta\left(x_{c}, \tau\right)=\pi \bar{\alpha}^{2} \tau^{2} / x_{c} \gg 1$. That is, in this limit, the spectrum takes the form in eq. (3.21) for any $x \leq x_{c}$. This large-time limit is merely formal, since, as already mentioned, the maximal value for $\zeta\left(x_{c}, \tau\right)$ which is physically allowed is $\zeta\left(x_{c}, \tau_{L}\right)=2 \pi \bar{\alpha}^{2}$, which is not that large. Still, this limit is conceptually interesting, in that it corresponds to the more familiar turbulence set-up: a steady situation in which the whole energy injected by the source flows through the spectrum into the 'sink' at $x=0$ (see the discussion in the next subsection).

It is finally interesting to clarify the suitability of perturbation theory (by which we mean the iterative solution to eq. (3.8) in which the branching term $\bar{\alpha} \mathcal{I}\left[D_{\text {rad }}\right]$ is treated as a small perturbation) for the problem at hand. Via successive iterations, one can construct a perturbative solution for $D_{\text {rad }}(x, \tau)$ in the form of a series in powers of $\bar{\alpha} \tau$ and is interesting to compare this series to the small- $\zeta$ expansion of the exact solution in eq. (3.20). Clearly, we do not expect this perturbative approach to be reliable near the endpoint of the spectrum at $x_{c}$, but one may hope that it becomes meaningful for $x$ well below $x_{c}$ and for small times $\bar{\alpha} \tau \ll 1$ - that is, in the region where the expansion (3.20) can be viewed too as a series in powers of $\bar{\alpha} \tau$. But even this last expectation is naive, as shown by the following argument: a perturbative solution via iterations would generate both odd and even powers of $\bar{\alpha} \tau$, whereas the corresponding expansion in eq. (3.20) contains only odd powers.

To further clarify this mismatch, we shall construct in appendix A the perturbative solution to low orders: $D_{\text {rad }}=D_{\text {rad }}^{(0)}+D_{\text {rad }}^{(1)}+D_{\text {rad }}^{(2)}+\cdots$. The zeroth order result is, clearly, $D_{\text {rad }}^{(0)}=\bar{\alpha} \tau / \sqrt{x}$, while the first iteration, as obtained by evaluating the branching term $\bar{\alpha} \mathcal{I}\left[D_{\text {rad }}\right]$ with the zeroth order result, yields precisely the correction of $\mathcal{O}(\bar{\alpha} \tau)$ shown in eq. (3.20), that is,

$$
D_{\operatorname{rad}}^{(1)}(x, \tau)=-\frac{\bar{\alpha}^{2} \tau^{2}}{\sqrt{x\left(x_{c}-x\right)}} .
$$

But a subtle issue shows up starting with the second iteration: the first-order correction $D_{\text {rad }}^{(1)}$ turns out to be an exact fixed point of the branching kernel: $\mathcal{I}\left[D_{\text {rad }}^{(1)}\right]=0$. Accordingly, the second-order correction is exactly zero, $D_{\text {rad }}^{(2)}=0$ (still in agreement with eq. (3.20)), but then the same is true for all the subsequent iterations: $D_{\text {rad }}^{(n)}=0$ for any $n \geq 2$. That 
is, the perturbative expansion, as computed without any approximation, terminates after just one non-trivial iteration and predicts $D_{\text {rad }}=D_{\text {rad }}^{(0)}+D_{\text {rad }}^{(1)}$. This prediction is certainly incorrect (except as an approximation at small times and small $x$ ): it differs from the actual expansion eq. (3.20) of the exact result and, in particular, it becomes negative and divergent when $x \rightarrow x_{c}$.

The mathematical origin of this failure will be clarified in appendix A. But its physical origin should be quite clear: we have already noticed the non-perturbative nature of the dynamics associated with the emission of very soft quanta, with energy fractions $x \lesssim$ $x_{s}=\bar{\alpha}^{2} \tau^{2}$. For such emissions, the effects of multiple branchings must be resumed to all orders and cannot be accurately studied via iterations. This non-perturbative dynamics is responsible for the rapid broadening of the LP peak and also for the fact that the radiation spectrum in eq. (3.12) exactly vanishes as $x \rightarrow x_{c}$ for any $\tau$. Similar, nonperturbative aspects affect the spectrum at any value of $x$, including the intermediate bins at $x_{s} \ll x \ll x_{c}$, since the occupation of any such a bin can change via the emission of very soft gluons. Hence, not surprisingly, the spectrum $D(x, \tau)$ cannot be faithfully computed within perturbation theory for generic values $(x, \tau)$, albeit interesting information can be obtained via this method in special cases, as we shall see.

\subsection{The energy flux}

As in section 2, the dissipative properties of the cascade, in particular, the rate for energy loss towards the medium, can be best studied by computing the energy flux associated with branchings. Let $\mathcal{E}\left(x_{0}, x_{c}, \tau\right)$ denote the energy which at time $\tau$ is contained in the modes in the spectrum within the interval $x_{0}<x<x_{c}$ :

$$
\mathcal{E}\left(x_{0}, x_{c}, \tau\right)=\int_{x_{0}}^{x_{c}} \mathrm{~d} x D_{\operatorname{rad}}(x, \tau) .
$$

When increasing $\tau$, this energy can change via two mechanisms: (i) it increases due to additional radiation by the source, at a rate $\int_{x_{0}}^{x_{c}} \mathrm{~d} x \mathcal{S}_{0}(x)$, and (ii) it decreases due to the energy transfer towards the modes at $x<x_{0}$ via gluon branching, at a rate which is by definition the energy flux $\mathcal{F}\left(x_{0}, \tau\right)$ through the bin $x_{0}$. Hence, we can write

$$
\frac{\partial \mathcal{E}\left(x_{0}, x_{c}, \tau\right)}{\partial \tau}=\int_{x_{0}}^{x_{c}} \mathrm{~d} x \mathcal{S}_{0}(x)-\mathcal{F}\left(x_{0}, \tau\right)
$$

which immediately implies

$$
\mathcal{F}\left(x_{0}, \tau\right)=\int_{x_{0}}^{x_{c}} \mathrm{~d} x \frac{\partial}{\partial \tau} \delta D_{\mathrm{br}}(x, \tau)=-\bar{\alpha} \int_{x_{0}}^{x_{c}} \mathrm{~d} x \mathcal{I}\left[D_{\mathrm{rad}}\right](x, \tau),
$$

where the first equality follows after recalling the definition (3.15) of $\delta D_{\mathrm{br}}(x, \tau)$, and the second one after also using the rate equation (3.8). Each of the two integral representations for $\mathcal{F}\left(x_{0}, \tau\right)$ in the equation above has its own virtues. When combined with the explicit result for $\delta D_{\mathrm{br}}(x, \tau)$ shown in eq. (3.15), the first representation allows for efficient numerical calculations, with results that we shall shortly describe. On the other hand, this formula is not so well suited for analytic studies, as we shall see. The second integral representation, 
which involves the branching term $\mathcal{I}\left[D_{\text {rad }}\right]$, is more directly connected to the dynamics of branchings and admits a transparent physical interpretation, to be discussed in section 3.4. A priori, this representation seems to be mathematically more involved, in that it involves a double convolution over the spectrum. Yet, as we shall see, this representation allows for more accurate analytic studies. In particular, it provides an exact analytic result in the important limit $x_{0} \rightarrow 0$.

Using the first equality in eq. (3.25) together with the expression (3.15) for $\delta D_{\mathrm{br}}(x, \tau)$, one finds, after simple manipulations,

$$
\mathcal{F}\left(x_{0}, \tau\right)=\frac{\bar{\alpha}}{\sqrt{\pi}} \int_{x_{0}}^{x_{c}} \frac{\mathrm{d} x}{\sqrt{x}} \gamma\left(\frac{1}{2}, \zeta\right),
$$

with $\zeta \equiv \zeta\left(x_{c}-x, \tau\right)$ as defined in eq. (3.13). We are mostly interested in the limit $x_{0} \rightarrow 0$ of this result, which represents the energy flux carried by the turbulent flow:

$$
\mathcal{F}_{\text {flow }}(\tau)=\frac{\bar{\alpha}}{\sqrt{\pi}} \int_{0}^{x_{c}} \frac{\mathrm{d} x}{\sqrt{x}} \gamma\left(\frac{1}{2}, \zeta\right) .
$$

As explained in section 2, this is the rate at which the energy leaks out of the spectrum and accumulates into a condensate at $x=0$. It is straightforward to numerically compute the integral in eq. (3.27) and thus study the flow as a function of $\tau$ for various values $x_{c} \ll 1$. The results are shown in figure 6 , together with the respective prediction of the 'low-energy' case $x_{c}>1$, that is, the function $\mathcal{F}_{\text {flow }}(\tau)$ in eq. (2.20). In principle, one should consider these curves only for $\tau$ values within the physically allowed range, i.e. for $\tau \leq \tau_{L}=\sqrt{2 x_{c}}$. But in figure 6 we also show them for larger values $\tau>\tau_{L}$; this is interesting too, but for a different physical problem (see below).

By comparing curves which refer to different values of $x_{c}$, one can better appreciate the role of the kinematical constraint $x \leq x_{c} \ll 1$ in slowing down the branching process and thus reducing the energy flow. The plots in figure 6 make clear that, when lowering $x_{c}$, one reduces not only the total duration $\tau_{L}$ of the branching process, but also the rate for energy loss at any given time $\tau<\tau_{L}$. This trend is natural on physical grounds: by decreasing $x_{c}$, one limits the phase-space for medium-induced radiation to emissions which carry lower and lower fractions of the total energy of the leading particle. Figure 6 also shows that the deviation between curves corresponding to different values of $x_{c}$ increases with time; for $\tau \sim \tau_{L}$, this deviation is seen to be sizable for all values of $x_{c}$ under consideration.

It would be interesting to understand the systematics of these plots via analytic studies. To that aim, one may attempt a small- $\tau$ expansion of the flow in eq. (3.27) based on the corresponding expansion of $\delta D_{\mathrm{br}}(x, \tau)$ in eq. (3.20). (This is tantamount to performing the small- $\zeta$ expansion of the function $\gamma(1 / 2, \zeta)$ in eq. (3.27).) At leading order, one should use the dominant contribution to $\delta D_{\mathrm{br}}(x, \tau)$, that is, (minus) the function $D_{\mathrm{rad}}^{(1)}(x, \tau)$ in eq. (3.22). One thus finds

$$
\mathcal{F}_{\text {flow }}(\tau) \simeq-\int_{0}^{x_{c}} \mathrm{~d} x \frac{\partial}{\partial \tau} D_{\text {rad }}^{(1)}(x, \tau)=2 \bar{\alpha}^{2} \tau \int_{0}^{x_{c}} \frac{\mathrm{d} x}{\sqrt{x\left(x_{c}-x\right)}}=2 \pi \bar{\alpha}^{2} \tau .
$$

This estimate, which is independent of $x_{c}$, holds only for sufficiently small times, such that $\zeta\left(x_{c}, \tau\right)=\pi \bar{\alpha}^{2} \tau^{2} / x_{c} \ll 1$, where it describes indeed the common behavior of all the curves 


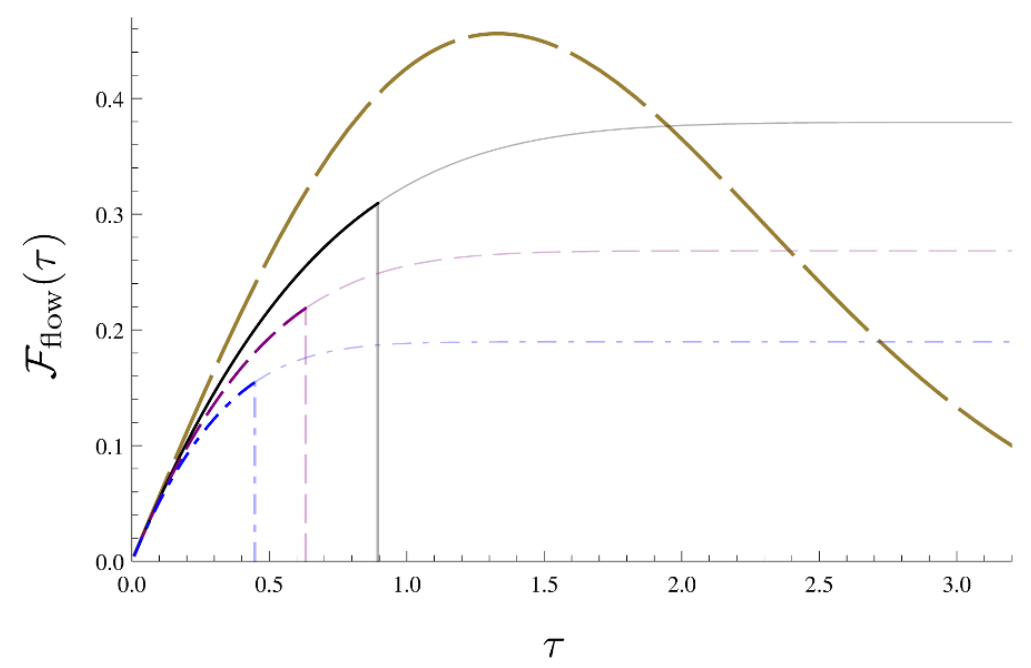

Figure 6. The rate of flow $\mathcal{F}_{\text {flow }}(\tau)$ as a function of $\tau$ for various physical regimes. The brown, long-dashed curve represents the function in eq. (2.20), which corresponds to $x_{c}>1$, that is, to the low energy regime. The other curves correspond to different values $x_{c}<1$ (i.e. to various 'highenergy' regimes) and are obtained according to eq. (3.27): $x_{c}=0.4$ (black, solid), $x_{c}=0.2$ (purple, dashed), and $x_{c}=0.1$ (blue, dashed-dotted). The thick lines represent the respective curves within their physical range of validity $\left(\tau<\tau_{L}\right)$, whereas the thin curves are their extrapolations at larger times $\tau>\tau_{L}$. The vertical lines denote the upper time limit $\tau_{L}=\sqrt{2 x_{c}}$.

exhibited in figure 8. But this approximation is unable to capture the lift in degeneracy with increasing $\tau$. One may expect to be able to compute corrections to eq. (3.28) by using the higher order terms in the expansion (3.20) of $D_{\text {rad }}$, but this turns out not to be possible: for all the terms in this expansion beyond $D_{\text {rad }}^{(1)}$, the integral over $x$ in eq. (3.27) develops a non-integrable singularity at its upper endpoint $x_{c}$.

In the next subsection, we shall exploit the second equality in eq. (3.25) to deduce an exact, analytic, result for $\mathcal{F}_{\text {flow }}(\tau)$ (see eq. (3.39)). But for the purposes of the present discussion, it suffices to consider just one more term in the small- $\tau$ expansion of $\mathcal{F}_{\text {flow }}(\tau)$. This can be obtained by expanding the exact result in eq. (3.39) and reads

$$
\mathcal{F}_{\text {flow }}(\tau) \simeq 2 \pi \bar{\alpha}^{2} \tau\left(1-\frac{\bar{\alpha} \tau}{\sqrt{x_{c}}}\right)
$$

As expected, the corrective term above lifts the degeneracy between different values of $x_{c}$. The relative importance of this term increases with time and becomes independent of $x_{c}$ when $\tau \sim \tau_{L}$ ( since $\tau_{L}$ itself scales like $\sqrt{x_{c}}$ ): $\bar{\alpha} \tau_{L} / \sqrt{x_{c}}=\sqrt{2} \bar{\alpha}$. Hence, this correction would be negligible in the formal weak coupling limit, but it is numerically important for realistic values of $\bar{\alpha}$ : e.g. $\sqrt{2} \bar{\alpha} \simeq 0.4$ for $\bar{\alpha}=0.3$. And indeed, the inclusion of this correction greatly improves the accuracy of the small-time expansion, as it will be shown later, in figure 8: the limited expansion in eq. (3.29) provides an excellent approximation to the exact result for any $\tau \leq \tau_{L}$.

Consider now the behavior of the flow for relatively large times $\tau \gg \tau_{L}$, that is, outside of the physical range for jet evolution. This corresponds to a different physical 
problem, which is closer to the familiar turbulence set-up - a steady source acts for arbitrarily large time and eventually builds up a time-independent energy spectrum -, except that our source has a rather unusual spectrum: rather than being localized near $x_{c}$ (e.g. $\mathcal{S}(x)=\delta\left(x-x_{c}\right)$ ), the function $\mathcal{S}_{0}(x)=\bar{\alpha} / \sqrt{x}$ has a long tail at small $x \leq x_{c}$, as expected for radiation. The associated steady flow at large times can be obtained as follows: from section 3.2 we recall that, when $\pi \bar{\alpha}^{2} \tau^{2} / x_{c} \gg 1$, the spectrum reaches the steady shape in eq. (3.21) (see also figure 5). From that moment on, the energy contained in the spectrum cannot increase anymore. For this to be possible, the energy flux associated with branchings must precisely equilibrate the rate for energy injection by the source; that is, the r.h.s. of eq. (3.24) must vanish:

$$
\mathcal{F}\left(x_{0}, \tau\right) \simeq \int_{x_{0}}^{x_{c}} \mathrm{~d} x \mathcal{S}_{0}(x)=2 \bar{\alpha}\left(\sqrt{x_{c}}-\sqrt{x_{0}}\right) .
$$

As expected, this result is independent of time and fixed by the source. For $x_{0}=0$, it yields

$$
\mathcal{F}_{\text {flow }}(\tau) \simeq 2 \bar{\alpha} \sqrt{x_{c}} \text { when } \pi \bar{\alpha}^{2} \tau^{2} / x_{c} \gg 1,
$$

which is indeed consistent with both the numerical results in figure 8 and the large-time asymptotics of eq. (3.27), as one can easily check. ${ }^{6}$

For comparison, let us also notice the spectrum and flux that would be generated by a localized source $\mathcal{S}(x)=A \delta\left(x-x_{c}\right)$ acting at $\tau \geq 0$. (This problem has been already considered in ref. [16].) For generic $\tau$, the corresponding spectrum coincides (up to a factor of $A$ ) with the r.h.s. of eq. (3.11) evaluated at $x_{1}=x_{c}$. For large times $\pi \bar{\alpha}^{2} \tau^{2} / x_{c} \gg 1$, this reaches the steady shape

$$
D_{\text {as }}(x)=\frac{A}{2 \pi \bar{\alpha}} \sqrt{\frac{x_{c}}{x\left(x_{c}-x\right)}} .
$$

In the same limit, the energy flux is steady and strictly uniform, $\mathcal{F}_{\text {as }}\left(x_{0}\right)=A$, as in standard turbulence. For $x \ll x_{c}$, these results are consistent with the Kolmogorov-Obukhov relation (2.21).

It is finally interesting to study the $x_{0}$-dependence of the energy flux in this high-energy case. This is expressed by eq. (3.26) that we have plotted in figure 7 as a function of $x_{0}$ for different values of $\tau$ and for $x_{c}=0.2$. Good analytic approximations can also be obtained. For relatively small times $\pi \bar{\alpha}^{2} \tau^{2} / x_{c} \ll 1$, and for $x_{0}$ not too close to $x_{c}$, it is convenient to rewrite eq. (3.26) as

$$
\mathcal{F}\left(x_{0}, \tau\right)=\mathcal{F}_{\text {flow }}(\tau)-\frac{\bar{\alpha}}{\sqrt{\pi}} \int_{0}^{x_{0}} \frac{\mathrm{d} x}{\sqrt{x}} \gamma\left(\frac{1}{2}, \zeta\right) .
$$

When $\zeta \ll 1$, we can use the Taylor expansion of the function $\gamma(1 / 2, \zeta)$, which is rapidly converging. To the same accuracy as in eq. (3.29), i.e. to second order in $\bar{\alpha} \tau$, it is enough to use $\gamma(1 / 2, \zeta) \simeq 2 \sqrt{\zeta}$, which yields

$$
\mathcal{F}\left(x_{0}, \tau\right) \simeq 2 \pi \bar{\alpha}^{2} \tau\left\{1-\frac{\bar{\alpha} \tau}{\sqrt{x_{c}}}-\frac{2}{\pi} \arcsin \sqrt{\frac{x_{0}}{x_{c}}}\right\} .
$$

\footnotetext{
${ }^{6}$ At large times, one has $\zeta \gg 1$ for any $x$, hence one can approximate $\gamma(1 / 2, \zeta) \simeq \sqrt{\pi}$ within eq. (3.27).
} 


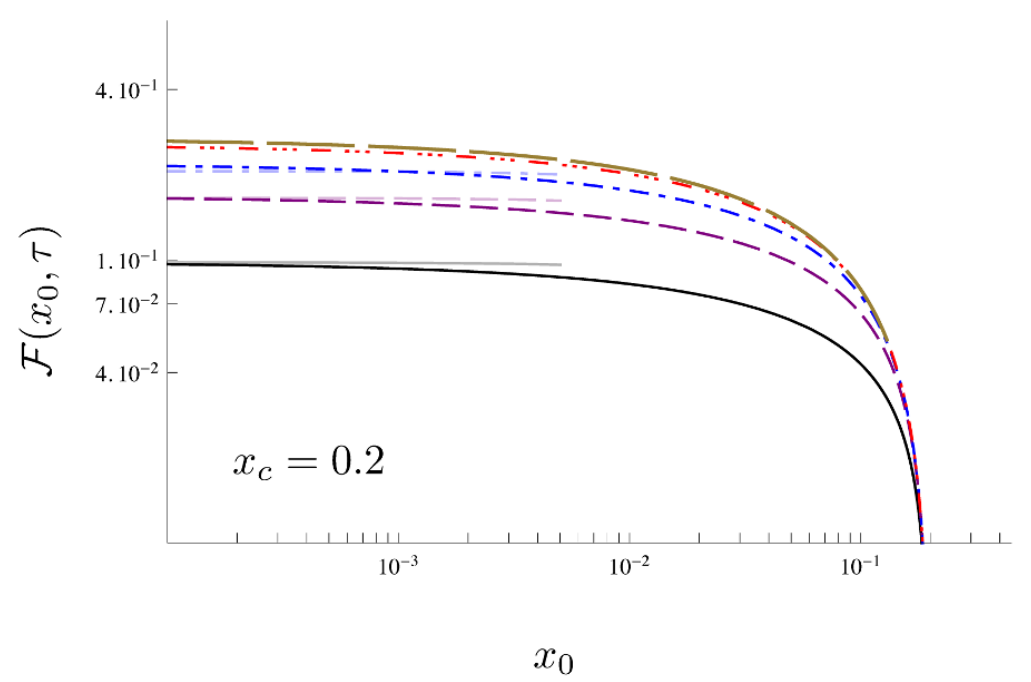

Figure 7. Plot (in log-log scale) of the energy flux $\mathcal{F}\left(x_{0}, \tau\right)$, cf. eq. (3.26), as a function of $x_{0}$ for $\bar{\alpha}=0.3, x_{c}=0.2$, and various values of $\tau: \tau=0.2$ (solid, black), $\tau=0.4$ (purple, dashed), $\tau=0.63$ (blue, dashed-dotted), $\tau=1$ (red, dashed-triple-dotted). The thin curves, shown for $\tau \leq \tau_{L}=0.63$ and $x_{0} \leq 0.005$, represent the approximation (3.34) valid at small $\tau$ and small $x_{0}$. The enveloping curve (brown, long-dashed) is the limiting curve at large $\tau$, cf. eq. (3.30).

At larger times $\pi \bar{\alpha}^{2} \tau^{2} / x_{c} \gg 1$, and also for $x_{0}$ very close to $x_{c}$ and any $\tau$, the flux takes the form in eq. (3.30). Both the numerical results in figure 7 and the analytic approximations in eqs. (3.34) and (3.30) demonstrate that the flux associated with branchings is quasiuniform (i.e. independent of $x_{0}$ ) for any $x_{0} \ll x_{c}$. As already mentioned, this signals a phenomenon of wave turbulence. Additional evidence in that sense will emerge from the analysis in the next subsection.

\subsection{The energy flux revisited: democratic branchings}

In this subsection, we shall present an alternative calculation of the energy flux, which exploits the second equality in eq. (3.25), i.e. the $x$-integral of the branching term $\mathcal{I}\left[D_{\text {rad }}\right]$. As we shall see, the main virtue of this alternative method is that it involves the gluon spectrum quasi-locally in $x$ : in order to compute the flux $\mathcal{F}\left(x_{0}, \tau\right)$ at small $x_{0} \ll x_{c}$, we need the spectrum $D_{\text {rad }}(x, \tau)$ at small $x \ll x_{c}$ as well. This property has important consequences, of both practical and conceptual nature. In practice, it will allow us to derive an exact analytic expression for the rate of flow $\mathcal{F}_{\text {flow }}(\tau)=\mathcal{F}\left(x_{0}=0, \tau\right)$ and to establish the analog of the Kolmogorov-Obhukov relation for the problem at hand. At a conceptual level, it sheds further light on the quasi-democratic nature of the in-medium branching process, which we recall is very unusual in the context of a gauge theory.

The integral of the branching term occurring in eq. (3.25) can be decomposed as

$$
-\int_{x_{0}}^{x_{c}} \mathrm{~d} x \mathcal{I}\left[D_{\mathrm{rad}}\right](x, \tau)=\int_{x_{0}}^{x_{c}} \mathrm{~d} x \mathcal{L}(x, \tau)+\int_{x_{0}}^{x_{c}} \mathrm{~d} x \mathcal{G}(x, \tau),
$$

where the two terms in the r.h.s. are the respective contributions of the 'loss' and 'gain' 
term in the rate equation. The 'loss' contribution is easily evaluated as

$$
\int_{x_{0}}^{x_{c}} \mathrm{~d} x \mathcal{L}(x, \tau)=\int_{0}^{1} \mathrm{~d} z z \mathcal{K}(z) \int_{x_{0}}^{x_{c}} \mathrm{~d} x \frac{D_{\operatorname{rad}}(x, \tau)}{\sqrt{x}} .
$$

In the 'gain' contribution, it is useful to change the integration variable as $x \rightarrow x^{\prime} \equiv x / z$ :

$$
\begin{aligned}
\int_{x_{0}}^{x_{c}} \mathrm{~d} x \mathcal{G}(x, \tau) & =-\int_{x_{0}}^{x_{c}} \mathrm{~d} x \int \mathrm{d} z \Theta\left(z-\frac{x}{x_{c}}\right) \mathcal{K}(z) \sqrt{\frac{z}{x}} D_{\operatorname{rad}}\left(\frac{x}{z}, \tau\right) \\
& =-\int \mathrm{d} z z \mathcal{K}(z) \Theta\left(z-\frac{x_{0}}{x_{c}}\right) \int_{x_{0} / z}^{x_{c}} \mathrm{~d} x^{\prime} \frac{D_{\operatorname{rad}}\left(x^{\prime}, \tau\right)}{\sqrt{x^{\prime}}}
\end{aligned}
$$

where, in the second line, the upper limit $x_{c}$ on $x^{\prime}$ follows from the condition $z>x / x_{c}$; also, the last $\Theta$-function, which enforces $z>x_{0} / x_{c}$, guarantees that the lower limit $x_{0} / z$ in the integral over $x^{\prime}$ remains smaller than the upper limit $x_{c}$. As usual, the 'gain' and 'loss' contributions taken separately develop singularities from the endpoint at $z=1$ of the integral over $z$, but these singularities cancel in the sum of the two contributions. Hence, the overall result is well defined and reads

$$
\begin{aligned}
\mathcal{F}\left(x_{0}, \tau\right)=\bar{\alpha} \int_{x_{0} / x_{c}}^{1} \mathrm{~d} z & z \mathcal{K}(z) \int_{x_{0}}^{x_{0} / z} \mathrm{~d} x \frac{D_{\operatorname{rad}}(x, \tau)}{\sqrt{x}} \\
& +\bar{\alpha} \int_{0}^{x_{0} / x_{c}} \mathrm{~d} z z \mathcal{K}(z) \int_{x_{0}}^{x_{c}} \mathrm{~d} x \frac{D_{\operatorname{rad}}(x, \tau)}{\sqrt{x}} .
\end{aligned}
$$

To better appreciate the physical interpretation of this result, let us return to the individual, 'loss' and 'gain', contributions, as shown in eq. (3.36) and respectively eq. (3.37).

The interpretation of the 'loss' term in eq. (3.36) is quite clear: this is the energy transferred per unit time from one parton generation to the next one via the branching of any of the 'hard' modes with $x_{0}<x<x_{c}$. (Recall that $\mathcal{K}(z) / \sqrt{x}$ represents the splitting rate for the parent mode $x$ into daughter modes $z x$ and $(1-z) x$. Also the factor of $z$ within the first integral can be equivalently replaced by $z \rightarrow[z+(1-z)] / 2=1 / 2$, due to the symmetry property $\mathcal{K}(z)=\mathcal{K}(1-z)$; hence, this factor truly accounts for the contribution of both daughter gluons.) However, some of these splittings do not contribute to the energy flux at $x_{0}$ : this is the case for the splittings with $z x>x_{0}$ (a condition which can be satisfied only for $z$ values which are large enough, namely $z>x_{0} / x_{c}$ ), for which the daughter gluons are still harder than $x_{0}$. The contributions of these splittings are therefore subtracted by the 'gain' term in eq. (3.37), which is negative indeed. Accordingly, the net result is the sum of two types of contributions, represented by the two terms in the r.h.s. of eq. (3.38): (i) relatively hard splittings with $x_{0} / x_{c}<z<1$, but such the parent gluon $x$ was close enough to $x_{0}$ (within the strip at $x_{0}<x<x_{0} / z$ ), and (ii) relatively soft splittings with $z<x_{0} / x_{c}$, in which case the parent gluon can be located anywhere between $x_{0}$ and $x_{c}$.

In the limit where $x_{0} \ll x_{c}$, the first term in the r.h.s. of eq. (3.38) dominates over the second one and controls the rate of flow. This is clear from the fact that the second term in eq. (3.38) vanishes when $x_{0} \rightarrow 0$, while the first one preserves a finite value in that limit, as we shall shortly see. Furthermore, still for $x_{0} \ll x_{c}$, the second term is controlled by 
very asymmetric splittings $\left(z<x_{0} / x_{c} \ll 1\right)$, whereas the first one is dominated by quasidemocratic branchings, that is, by generic $z$ values in the bulk, which are not specially close to either the lower limit $z=x_{0} / x_{c} \ll 1$, or the upper limit $z=1$, of the $z$-integral. Indeed, this integral is rapidly convergent both at small $z$, because of the factor of $z$ in the integrand, and at $z \rightarrow 1$, because the result of the integral over $x$ linearly vanishes in that limit.

The dominance of quasi-democratic branchings at small $x_{0}$ allows us to construct an exact solution for the energy flux in the limit $x_{0} \rightarrow 0$ and for the simplified kernel $\mathcal{K}_{0}(z)$ (for which the spectrum is analytically known). Specifically, the fact that the integral over $z$ within the first term in eq. (3.38) is not specially sensitive to its lower limit $x_{0} / x_{c}$ means that the relevant values of $z$ do not scale like $x_{0}$ when $x_{0} \rightarrow 0$. Hence, the upper limit $x_{0} / z$ of the integral over $x$ vanishes when $x_{0} \rightarrow 0$, so like the corresponding lower limit. Accordingly, this integral is controlled by very small values of $x$, which $d o$ scale like $x_{0}$ and in particular are much smaller than $x_{c}$. It is then justified to evaluate this integral using the dominant behavior of the spectrum for $x \ll x_{c}$, that is, eq. (3.18). With this scaling behavior $\sim 1 / \sqrt{x}$, the integral over $x$ is logarithmic and its result is independent of $x_{0}$. One thus finds

$$
\mathcal{F}_{\text {flow }}(\tau)=2 \pi \bar{\alpha}^{2} \tau\left[1-h\left(\zeta_{0}\right)\right], \quad \zeta_{0} \equiv \zeta\left(x_{c}, \tau\right)=\frac{\pi \bar{\alpha}^{2} \tau^{2}}{x_{c}},
$$

where the overall factor $2 \pi$ has been generated as

$$
2 \pi=\int_{0}^{1} \mathrm{~d} z z \mathcal{K}_{0}(z) \ln \frac{1}{z}=\int_{0}^{1} \mathrm{~d} z \frac{1}{\sqrt{z}(1-z)^{3 / 2}} \ln \frac{1}{z} .
$$

Using the properties of the function $h(\zeta)$ discussed in section 3.2, one can easily recover both the small $-\tau$ expansion of the flow, as anticipated in eq. (3.29), and its large $-\tau$ asymptotics in eq. (3.31). As a check of eq. (3.39), we display this result in figure 8 (as a function of $\tau$ for several values of $x_{c}$ ) versus the result of the numerical integration in eq. (3.26). One can also see in this figure that the limited expansion (3.29) is indeed a very good approximation for any $\tau$ in the physical range, as already noticed in section 3.3. This is understandable since the first correction beyond eq. (3.29) in the small- $\tau$ expansion of eq. (3.39) is exactly vanishing, as manifest on eq. (3.20).

By inspection of eqs. (3.18) and (3.39), it is obvious that the spectrum at small $x$ is proportional to the flow, in the sense of eq. (2.21). The above construction of eq. (3.39) explains the physical origin of this proportionality and also suggests that it is quite general: it holds for any splitting kernel with the singularity structure shown in eq. (2.6), since any such a kernel leads to democratic branchings and to a spectrum which at small $x$ has the shape of the scaling spectrum $D_{\mathrm{sc}}(x)=1 / \sqrt{x}$. The time dependence of the spectrum (again at small $x$ ) depends upon the detailed structure of the branching kernel (it is generally different for the full kernel $\mathcal{K}(z)$ and for the simplified one $\mathcal{K}_{0}(z)$ ), and also upon the nature of the 'source' at large $x$ (it is e.g. different for a source localized at $x_{c}, \mathcal{S}(x)=A \delta\left(x-x_{c}\right)$, as opposed to a radiation source $\left.\mathcal{S}_{0}(x)=\theta\left(x_{c}-x\right) \bar{\alpha} / \sqrt{x}\right)$. But the rate of flow $\mathcal{F}_{\text {flow }}(\tau)$ has exactly the same time-dependence as the spectrum and the proportionality relation (2.21) 


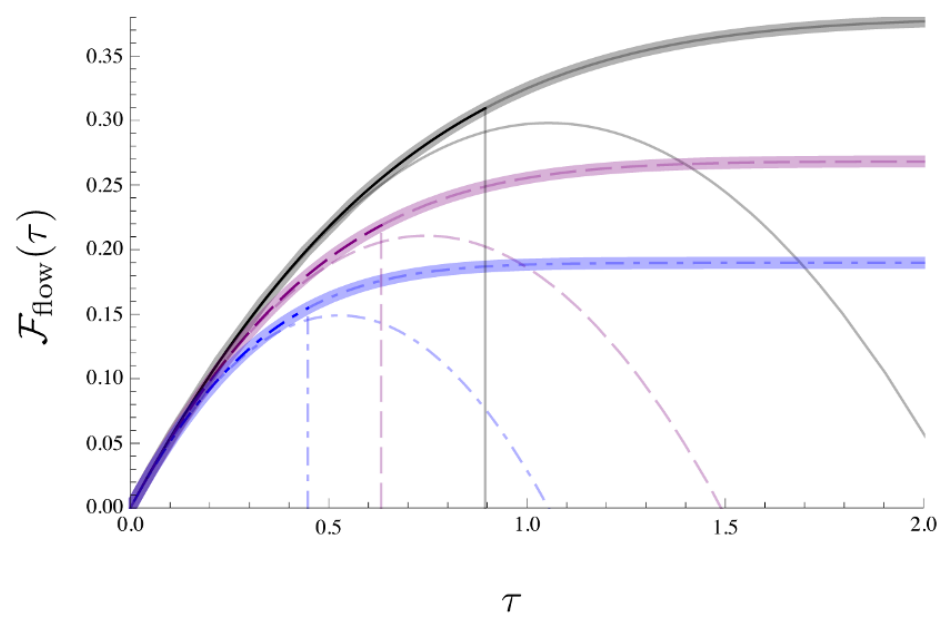

Figure 8. The rate of flow $\mathcal{F}_{\text {flow }}(\tau)$ as a function of $\tau$ in the high-energy regime for various values of $x_{c}: x_{c}=0.4$ (black, solid), $x_{c}=0.2$ (purple, dashed), and $x_{c}=0.1$ (blue, dashed-dotted). The thick lines represent the respective curves within their physical range of validity $\left(\tau<\sqrt{2 x_{c}}\right)$, as computed by numerical integration in eq. (3.26). The thin curves following the thick ones are the predictions of eq. (3.26) for larger times, outside the physical range $\left(\tau>\sqrt{2 x_{c}}\right)$. The thin curves deviating from the thick ones correspond to the limited expansion in eq. (3.29). Finally, the very thick (opaque) curves are the new, fully explicit, analytic result in eq. (3.39). The vertical lines denote the physical upper limit on time $\tau_{L}=\sqrt{2 x_{c}}$.

universally holds, with a proportionality factor which is however kernel-dependent:

$$
D(x, \tau) \simeq \frac{1}{v \bar{\alpha}} \frac{\mathcal{F}_{\text {flow }}(\tau)}{\sqrt{x}} \quad \text { for } \quad x \ll x_{c} .
$$

Here, $v$ is a pure number, defined by the obvious generalization of eq. (3.40):

$$
v \equiv \int_{0}^{1} \mathrm{~d} z z \mathcal{K}(z) \ln \frac{1}{z}=\int_{0}^{1} \mathrm{~d} z \frac{f(z)}{\sqrt{z}(1-z)^{3 / 2}} \ln \frac{1}{z} \simeq 4.96 .
$$

On both eq. (3.42) or eq. (3.40), it is obvious that the respective integral over $z$ is dominated by generic values in the bulk, as expected for quasi-democratic branchings. As discussed after eq. (2.15), $v$ has the physical interpretation of the average number of soft primary gluons with energies $\omega \sim \omega_{s}(t)=\bar{\alpha}^{2} \hat{q} t^{2} / 2$ that are emitted by the leading particle during a time $t$.

Eq. (3.41) is particularly useful in a steady situation, where the energy flux is a priori known, since determined by the external source. (This is the case in the familiar turbulence problem, where the Kolmogorov-Obhukov relation has been originally identified.) As a simple, yet non-trivial, application of this type, consider the steady situation reached when the external source $\mathcal{S}_{0}(x)=\theta\left(x_{c}-x\right) \bar{\alpha} / \sqrt{x}$ acts for sufficiently large time $\bar{\alpha}^{2} \tau^{2} \gg x_{c}$. The corresponding flow is given by eq. (3.31) and then eq. (3.41) can be used to deduce the asymptotic spectrum at large times and small $x$ :

$$
D(x, \tau \rightarrow \infty) \simeq \frac{2}{v} \sqrt{\frac{x_{c}}{x}} \quad \text { for } \quad x \ll x_{c} .
$$




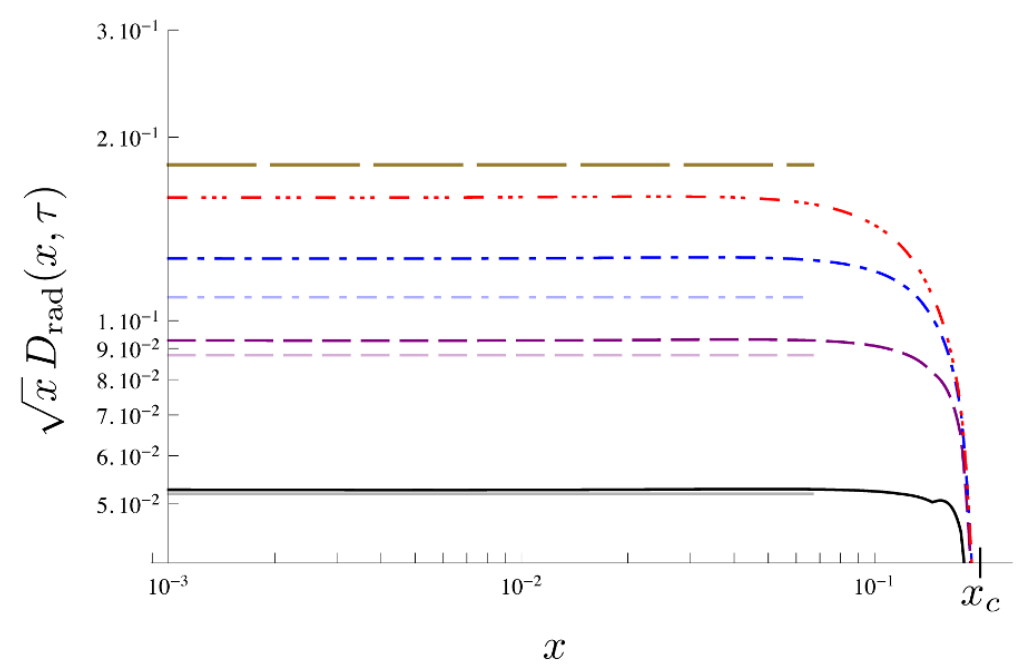

Figure 9. The numerical solution to the rate equation eq. (3.8) with the full splitting kernel $\mathcal{K}$ from eq. (2.6), for $x_{c}=0.2$ and various values of $\tau: \tau=0.2$ (solid, black), $\tau=0.4$ (purple, dashed), $\tau=0.63$ (blue, dashed-dotted), $\tau=1$ (red, dashed-triple-dotted). The thin curves, shown for $\tau \leq \tau_{L}=0.63$ and $x \leq 0.07$, represent the small $-\tau$ and small- $x$ approximation in eq. (3.44). The enveloping curve (brown, long-dashed) is the limiting curve at large $\tau$, cf. eq. (3.43).

This result is interesting in that it represents a non-perturbative prediction associated with the full kernel, for which exact analytic solutions are not known. (For the simplified kernel, $v \rightarrow 2 \pi$ and eq. (3.43) reduces to eq. (3.21), as it should.)

Still for the full kernel, eq. (3.41) can also be used in the reversed way, namely to deduce the flow from the spectrum in the small-time regime at $\bar{\alpha}^{2} \tau^{2} \ll x_{c}$. Indeed, in this limit and for $x \ll x_{c}$, the spectrum can be computed in perturbation theory, via iterations (see the discussion in appendix A). To second order in $\bar{\alpha} \tau$, the result turns out to be the same as for the simplified kernel $\mathcal{K}_{0}(z)$, namely (compare to eq. (3.20))

$$
D_{\mathrm{rad}}(x, \tau) \simeq \frac{\bar{\alpha} \tau}{\sqrt{x}}\left(1-\frac{\bar{\alpha} \tau}{\sqrt{x_{c}}}\right) \quad \text { for } \quad \bar{\alpha}^{2} \tau^{2} \ll x_{c} \quad \text { and } \quad x \ll x_{c} .
$$

By using this approximation together with eq. (3.41), we can obtain the generalization of eq. (3.29) to the case of the complete kernel:

$$
\mathcal{F}_{\text {flow }}(\tau)=v \bar{\alpha}^{2} \tau\left(1-\frac{\bar{\alpha} \tau}{\sqrt{x_{c}}}\right)
$$

This result is quite useful, in particular for phenomenology, in that it offers a good accurate estimate for the energy loss via flow for the case of the physical kernel. In figure 9 we show the numerical solution to the rate equation (3.8) with the full splitting kernel $\mathcal{K}$ in eq. (2.6), together with its analytic approximations valid at small $x$ : eq. (3.44) at small $\tau$ and eq. (3.43) at large $\tau$.

\section{Physical discussion: energy loss at large angles}

In this section, we summarize the results obtained in the previous sections and deduce some physical consequences which, modulo some assumptions, can be compared to the 
phenomenology of di-jet asymmetry at the LHC, at least qualitatively. Ultimately, we are interested in the energy transported at large angles with respect to the jet axis. To study this, we successively consider the three following quantities:

(I) the flow energy $\mathcal{E}_{\text {flow }}(\tau)$ : this is the energy fraction carried away by the turbulent flow and which formally ends up in a condensate at $x=0$;

(II) the thermalization energy $\mathcal{E}_{\mathrm{th}}(\tau)$ : this is the energy fraction which is carried by quanta with $x<x_{\text {th }} \equiv T / E$, which are assumed to thermalize and thus transmit their energy to the medium.

(III) the energy transported at angles larger than a given value $\theta_{0}$ : the definition of this quantity requires some additional discussion and is postponed after the study of the two previous ones.

(I). The flow energy can be calculated in two alternative ways: as the $\tau$-integral of the respective flux $\mathcal{F}_{\text {flow }}(\tau)$, which is explicitly given by eq. (3.39), or as the $x$-integral of the change $\delta D_{\text {br }}(x, \tau)$ in the spectrum due to branchings, as shown in eq. (3.15),

$$
\mathcal{E}_{\text {flow }}(\tau) \equiv \int_{0}^{\tau} \mathrm{d} \tau^{\prime} \mathcal{F}_{\text {flow }}\left(\tau^{\prime}\right)=\int_{0}^{x_{c}} \mathrm{~d} x \delta D_{\mathrm{br}}(x, \tau)
$$

The second representation relies on the fact that the flow energy is by definition the difference between the total energy supplied by the source $\mathcal{S}_{0}(x)$ and the energy which remains in the spectrum. Here, we shall use this second representation to numerically compute $\mathcal{E}_{\text {flow }}$, but rely on the first one for analytic estimates. Indeed, we know that the limited expansion of the flow rate shown in eq. (3.45) is rather accurate for any $\tau \leq \tau_{L}$; this can be easily integrated over time,

$$
\mathcal{E}_{\text {flow }}(\tau) \simeq \frac{v}{2} \bar{\alpha}^{2} \tau^{2}\left(1-\frac{2}{3} \frac{\bar{\alpha} \tau}{\sqrt{x_{c}}}\right)
$$

This estimate holds for the full kernel $\mathcal{K}(z)$, but the corresponding result for the simplified kernel $\mathcal{K}_{0}(z)$ is simply obtained by replacing $v \rightarrow 2 \pi$ in the prefactor.

In figure 10 we show the flow energy evaluated at the end of the evolution $(\tau=$ $\tau_{L}=\sqrt{2 x_{c}}$ ) as a function of $x_{c}$ and for two values of $\bar{\alpha}$. The exact respective results, cf. eq. (4.1), are compared with the limited expansion in eq. (4.2) (which is seen to be accurate, as expected) and with the prediction (2.14) of the 'low-energy case' extrapolated to $x_{c} \ll 1$, that is, outside its physical range of validity. The purpose of this extrapolation is to emphasize that, by ignoring the kinematical constraint $x \leq x_{c}$, one would significantly overestimate the energy loss via flow.

Remarkably, figure 10 shows that the quantity $\mathcal{E}_{\text {flow }}\left(\tau_{L}\right)$ is a linear function of $x_{c}$. This property is obvious for the limited expansion in eq. (4.2), but is in fact exact within the present effective theory too: using eq. (3.15) for $\delta D_{\mathrm{br}}(x, \tau)$, we write

$$
\mathcal{E}_{\text {flow }}\left(\tau_{L}\right)=\bar{\alpha} \tau_{L} \int_{0}^{x_{c}} \frac{\mathrm{d} x}{\sqrt{x}} h\left(\frac{\pi \bar{\alpha}^{2} \tau_{L}^{2}}{x_{c}-x}\right)=\sqrt{2} \bar{\alpha} x_{c} \int_{0}^{1} \frac{\mathrm{d} u}{\sqrt{u}} h\left(\frac{2 \pi \bar{\alpha}^{2}}{1-u}\right),
$$




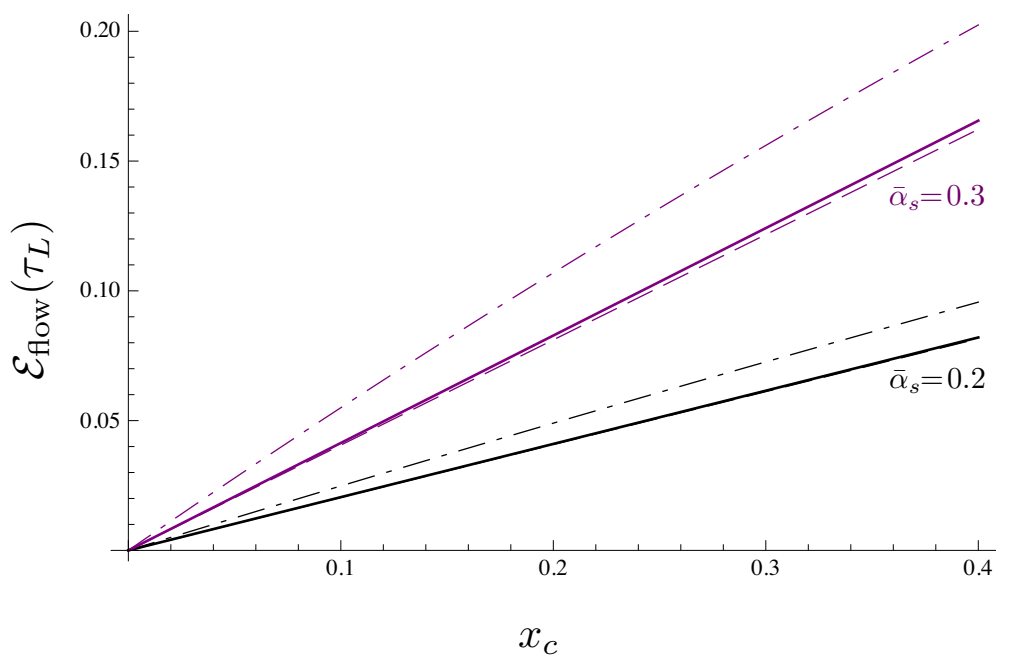

Figure 10. The energy fraction $\mathcal{E}_{\text {flow }}\left(\tau_{L}\right)$ carried by the turbulent flow, i.e. eq. (4.1) with $\tau=$ $\tau_{L} \equiv \sqrt{2 x_{c}}$, plotted as a function of $x_{c}$ for two values of the coupling constant: $\bar{\alpha}=0.2$ (black) and $\bar{\alpha}=0.3$ (purple). Solid lines: the exact result obtained by numerical integration in the second equality in eq. (4.1). Dashed lines: the weak-coupling expansion in eq. (4.2), that is, $\mathcal{E}_{\text {flow }}=2 \pi \bar{\alpha}^{2} x_{c}(1-2 \sqrt{2} \bar{\alpha} / 3)$. (For $\bar{\alpha}=0.2$, this approximation can hardly be distinguished from the exact curve.) For comparison, we also show, with dashed-dotted lines, the respective predictions of the 'low-energy case', i.e. eq. (2.14) with $\tau=\sqrt{2 x_{c}}$.

where the r.h.s. is linear in $x_{c}$, as anticipated. This is interesting as it implies that the energy which is carried away by the flow, namely (cf. eq. (4.2)),

$$
\Delta E_{\text {flow }} \equiv E \mathcal{E}_{\text {flow }}\left(\tau_{L}\right) \simeq v \bar{\alpha}^{2} \omega_{c}\left(1-\frac{2 \sqrt{2}}{3} \bar{\alpha}\right)
$$

is independent of the energy $E$ of the leading particle and parametrically of order $\bar{\alpha}^{2} \omega_{c}=\omega_{s}^{2}$ (the natural energy scale for multiple branchings). One should however keep in mind that this conclusion holds only for sufficiently energetic jets, such that $x_{c} \ll 1$, or $E \gg \omega_{c}$. Notice also that the energy loss in eq. (4.4) is enhanced by the relatively large numerical factor $v(1-2 \sqrt{2} \bar{\alpha} / 3)(\simeq 3.5$ for $\bar{\alpha}=0.3)$ as compared to its parametric estimate $\bar{\alpha}^{2} \omega_{c}$. This is mostly due to the factor $v \simeq 4.96$, which we recall is the average number of soft primary emissions with energies $\omega \sim \omega_{s}$.

(II). Given the flow energy in eq. (4.1), the thermalization energy can immediately be computed as the sum between $\mathcal{E}_{\text {flow }}(\tau)$ and the energy contained in the bins of the spectrum at $x \leq x_{\text {th }}$ :

$$
\mathcal{E}_{\text {th }}(\tau)=\mathcal{E}_{\text {flow }}(\tau)+\int_{0}^{x_{\text {th }}} \mathrm{d} x D_{\text {rad }}(x, \tau)
$$

In practice, $x_{\text {th }} \ll x_{c}$, which allows us to approximate the above integral as

$$
\mathcal{E}_{\mathrm{th}}(\tau) \simeq \frac{v}{2} \bar{\alpha}^{2} \tau^{2}\left(1-\frac{2}{3} \frac{\bar{\alpha} \tau}{\sqrt{x_{c}}}\right)+2 \bar{\alpha} \tau \sqrt{x_{\mathrm{th}}}\left(1-\frac{\bar{\alpha} \tau}{\sqrt{x_{c}}}\right)
$$




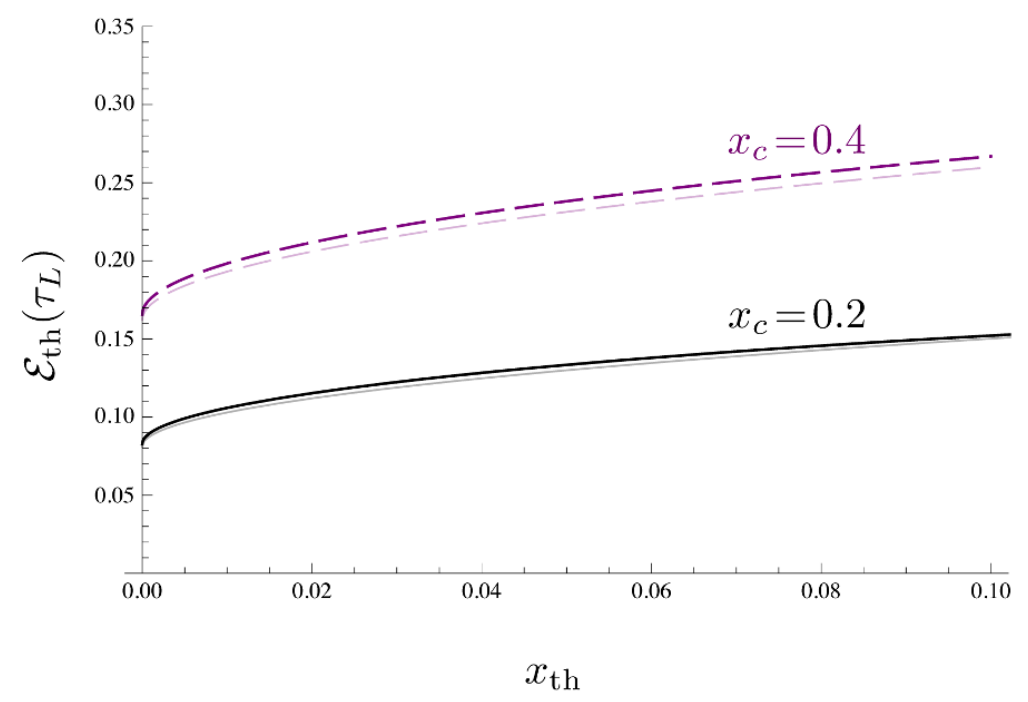

Figure 11. The energy fraction which 'thermalizes' $\mathcal{E}_{\text {th }}\left(\tau_{L}\right)$, plotted as a function of the thermalization scale $x_{\text {th }}$ for two values of $x_{c}$ : $x_{c}=0.4$ (black, solid) and $x_{c}=0.2$ (purple, dotted). The thick curves are the exact result obtained via numerical integration in eq. (4.5). The thin, opaque, curves are the respective predictions of the limited expansion in eq. (4.6) with $v \rightarrow 2 \pi$.

We emphasize that this result is fully obtainable from perturbation theory, including the complete kernel (2.6): it only requires the second iteration to the spectrum in eq. (3.44). As manifest in eq. (4.6), the flow contribution to $\mathcal{E}_{\text {th }}(\tau)$ is formally of higher order in $\bar{\alpha} \tau$, yet it dominates over the 'spectrum' contribution as soon as $x_{\text {th }}$ is small enough: for $\tau=\tau_{L}$, the flow dominates provided $x_{\mathrm{th}}<x_{s}=\bar{\alpha}^{2} x_{c}$ (or, equivalently, $T<\omega_{s}$ ), a condition which is well satisfied in practice (see below).

In figure 11 we plot $\mathcal{E}_{\mathrm{th}}\left(\tau_{L}\right)$ as a function of $x_{\mathrm{th}}$ for $x_{c}=0.2$ and $x_{c}=0.4$, and for the simplified kernel $\mathcal{K}_{0}$. The exact result as obtained via numerical integration in eq. (4.5) is compared to the limited expansion in eq. (4.6) with $v \rightarrow 2 \pi$.

(III). We now turn to the third quantity introduced above, namely the energy loss at large angles. Let $\mathcal{E}\left(\theta>\theta_{0}, \tau\right)$ denote the energy fraction which after a time $\tau$ has been transported to angles larger than a given value $\theta_{0}$. So far, we have considered only the energy distribution of the gluons in the cascade, but did not resolve their distribution in transverse momentum $\boldsymbol{k}$, or in the polar angle $\theta$ w.r.t. the jet axis (defined as $\sin \theta=k_{\perp} / \omega$ ). Rather, the $\boldsymbol{k}$-distribution has been explicitly integrated out, as shown in eq. (2.1), in order to obtain simpler versions for the rate equations. Yet, it turns out that for qualitative and even semi-quantitative estimates, one can restore the $\theta$-distribution via the following, simple, argument. All the gluons in the cascade which are not too soft (namely, those with $x \gtrsim x_{s}=\bar{\alpha}^{2} x_{c}$ ) propagate in the medium along a distance of order $L$ and hence accumulate via multiple scattering an average transverse momentum squared $\left\langle k_{\perp}^{2}\right\rangle \simeq Q_{L}^{2} \equiv \hat{q} L$, which is independent of $x$. As long as this momentum $Q_{L}$ is much smaller than the gluon energy $\omega=x E$, one can estimate the propagation angle according to

$$
\theta(x) \simeq \frac{Q_{L}}{x E}=\frac{x_{c}}{x} \theta_{c}, \quad \text { with } \quad \theta_{c} \equiv \frac{Q_{L}}{\omega_{c}}=\frac{2}{\sqrt{\hat{q} L^{3}}} .
$$


Hence, the interesting quantity $\mathcal{E}\left(\theta>\theta_{0}, \tau\right)$ can be computed as the energy fraction $\mathcal{E}^{<}\left(x_{0}, \tau\right)$ carried by the gluons with $x<x_{0}$, where $x_{0} \equiv x_{c}\left(\theta_{c} / \theta_{0}\right)$. This is of course the same as the 'thermalization energy' in eq. (4.5) evaluated for $x_{\mathrm{th}}=x_{0}$. Hence, plotting the following quantity

$$
\mathcal{E}^{<}\left(x_{0}, \tau\right) \equiv \mathcal{E}_{\text {flow }}(\tau)+\int_{0}^{x_{0}} \mathrm{~d} x D_{\text {rad }}(x, \tau)
$$

as a function of $x_{c} / x_{0}$ is tantamount to representing the quantity $\mathcal{E}\left(\theta>\theta_{0}, \tau\right)$ as a function of $\theta_{0} / \theta_{c}$. This is strictly true as long as the angle $\theta_{0}$ is not too large, namely $\theta_{0} \lesssim \theta_{c} / \bar{\alpha}^{2}$, in order for the condition $x_{0} \gtrsim x_{s}$ to remain satisfied. ${ }^{7}$ But as we argue now, this is not a serious limitation. Indeed, for smaller values $x_{0}<x_{s}$, the r.h.s. of eq. (4.8) is dominated by the flow piece, which is independent of $x_{0}$; accordingly, for $\theta_{0}$ larger than $\theta_{s} \equiv \theta\left(x_{s}\right) \simeq \theta_{c} / \bar{\alpha}^{2}$, the function $\mathcal{E}\left(\theta>\theta_{0}, \tau\right)$ is quasi-independent of $\theta_{0}$ and approximately equal to $\mathcal{E}_{\text {flow }}(\tau)$. An intuitive view of the angles $\theta_{c}$ and $\theta_{s}$ in the context of a typical gluon cascade is provided by figure 1 .

In practice, it is more convenient to plot the complementary quantity, namely the energy fraction located at $x$-values larger than $x_{0}$, meaning at angles $\theta$ smaller than a value $\theta_{0}=\theta_{c}\left(x_{c} / x_{0}\right)$ :

$$
\begin{aligned}
\mathcal{E}^{>}\left(x_{0}, \tau\right) \equiv 1-\mathcal{E}^{<}\left(x_{0}, \tau\right) & =\mathcal{E}_{\mathrm{LP}}(\tau)+\mathcal{E}\left(x_{0}, x_{c}, \tau\right) \\
& =1-2 \bar{\alpha} \tau \sqrt{x_{c}}+\int_{x_{0}}^{x_{c}} \mathrm{~d} x D_{\mathrm{rad}}(x, \tau) \\
& =1-2 \bar{\alpha} \tau \sqrt{x_{0}}-\int_{x_{0}}^{x_{c}} \mathrm{~d} x \delta D_{\mathrm{br}}(x, \tau) .
\end{aligned}
$$

Indeed, within our present set-up, the quantity $E_{J}\left(\theta_{0}\right) \equiv E \mathcal{E}^{>}\left(x_{0}, \tau_{L}\right)$ can be identified with the total jet energy for a jet with opening angle $\theta_{0}$. As emphasized by the second equality above, this is the sum of the energy $\mathcal{E}_{\mathrm{LP}}$ of the leading particle and that carried by the radiation modes at $x_{0}<x<x_{c}$. Vice-versa, the difference $\Delta E\left(\theta_{0}\right) \equiv E-E_{J}\left(\theta_{0}\right)=$ $E \mathcal{E}^{<}\left(x_{0}, \tau_{L}\right)$ is the energy lost by the jet, i.e. the energy which has been transported by the radiation to angles larger than $\theta_{0}$.

In figure 12, the quantity (4.9) is represented as a function of $x_{c} / x_{0}$ for $\tau=\tau_{L}$ and $x_{c}=0.4$. We also show the single-branching (or BDMPSZ) approximation, $\mathcal{E}^{>}\left(x_{0}, \tau_{L}\right)=$ $1-2 \bar{\alpha} \sqrt{2 x_{c} x_{0}}$, as obtained by neglecting the integral of $\delta D_{\mathrm{br}}$ in the third line of eq. (4.9). In addition, we plot the respective prediction of the low-energy case, eq. (2.17), which here is extrapolated outside its physical range.

Two features of these curves are worth emphasizing: first, the two curves which include the effects of multiple branchings (the solid curve and the dashed one) show an offset at large $x_{c} / x_{0}$, meaning that at large angles the difference $1-\mathcal{E}^{>}\left(x_{0}\right)=\mathcal{E}^{<}\left(x_{0}\right)$ approaches

\footnotetext{
${ }^{7}$ The softer gluons with $x \lesssim x_{s}$ have a shorter lifetime $\Delta t(x)<L$, as shown in eq. (2.22). The corresponding transverse momentum broadening is estimated as $\left\langle k_{\perp}^{2}\right\rangle(x) \sim \hat{q} \Delta t(x)$, and the relation (4.7) between the propagation angle $\theta(x)$ and the reference angle $\theta_{c}$ gets replaced by (to parametric accuracy) $\theta(x) / \theta_{c} \sim(1 / \sqrt{\bar{\alpha}})\left(x_{c} / x\right)^{3 / 4}[18-20]$.
} 


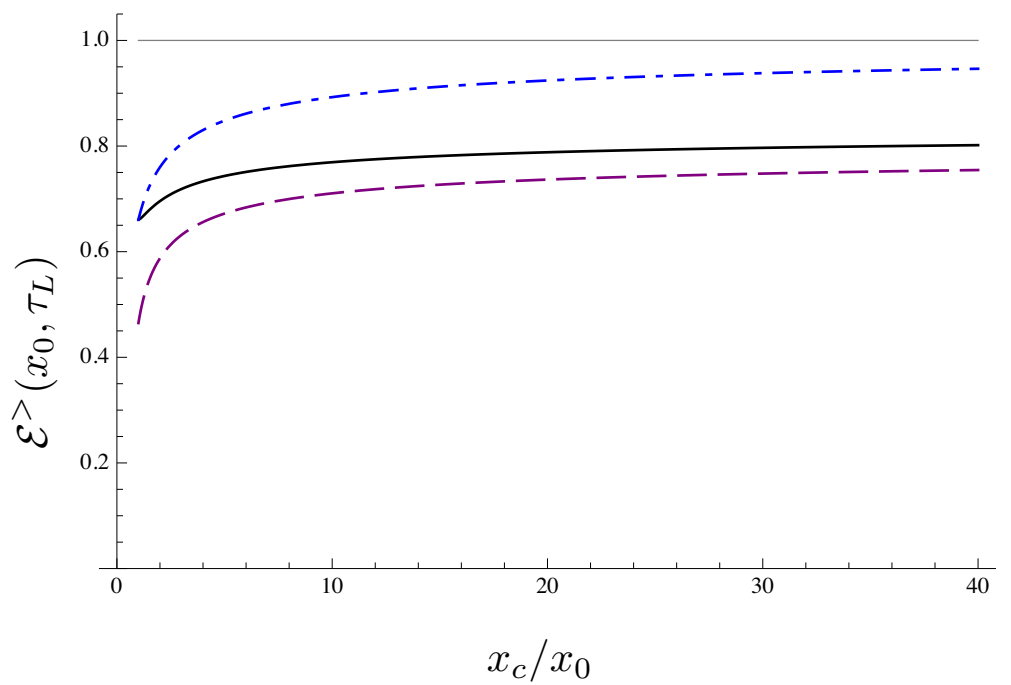

Figure 12. The energy $\mathcal{E}^{>}\left(x_{0}, \tau_{L}\right)$ contained in the bins of the spectrum with $x \geq x_{0}$ at the end of the evolution plotted as a function of $x_{c} / x_{0}$ for $x_{0} \leq x_{c}, x_{c}=0.4$, and $\bar{\alpha}=0.3$. Black, solid, curve: the full result computed according to eq. (4.9). Blue, dotted-dashed, curve: the approximation obtained by neglecting the effects of multiple branchings. Purple, dashed, curve: the respective prediction of the low-energy case, eq. (2.17), which is extrapolated to $x_{c}=0.4$. As explained in the text, these curves can also be viewed as representing the energy fraction $\mathcal{E}_{J}\left(\theta_{0}\right)$ contained within a jet with opening angle $\theta_{0}$ plotted as a function of $\theta_{0} / \theta_{c}$.

a finite value as $x_{c} / x_{0} \rightarrow \infty$. This non-zero value is, of course, the energy fraction $\mathcal{E}_{\text {flow }}$ taken away by the turbulent flow - the distinguished feature of the medium-induced multiple branching. As a check, we also notice from figure 12 that this offset is absent, as it should, for the corresponding prediction of the BDMPSZ spectrum. For applications to the phenomenology, it is interesting to observe that the kinematic restriction to $x<x_{c}$ (which applies whenever $x_{c}<1$ ) significantly reduces the value of this offset. This reduction is visible in both figure 12 and figure 10.

Second, as also visible in figure 12 (and anticipated after eq. (4.8)), the variation with $x_{c} / x_{0}$ is extremely slow, especially for the two curves which include the effects of multiple branchings. Physically, this means that, by increasing the jet opening angle $\theta_{0}=\left(x_{c} / x_{0}\right) \theta_{c}$, one can recover some of the energy that has been transported to large angles, but only very slowly. This is so because most of this energy has been transported, by the turbulent flow, directly at very large angles $\theta \gtrsim \theta_{\text {th }}$, where it has been lost towards the medium via thermalization. Here, $\theta_{\mathrm{th}}$ is the propagation angle for very soft quanta with $x \sim x_{\mathrm{th}}\left(\theta_{\mathrm{th}}\right.$ is significantly larger than $\theta_{s}$, since $x_{\mathrm{th}}$ is much smaller than $x_{s}=\bar{\alpha}^{2} x_{c}$ ). In principle, this angle $\theta_{\text {th }}$ can be estimated within our effective theory - to parametric accuracy one finds $\theta_{\mathrm{th}} / \theta_{c} \sim(1 / \sqrt{\bar{\alpha}})\left(x_{c} / x_{\mathrm{th}}\right)^{3 / 4}$, cf. footnote $7-$, but this estimate is questionable: the angular distribution of the very soft gluons with $x \sim x_{\text {th }}$ could be influenced by other effects, like the precise mechanism of thermalization, the Bethe-Heitler limit on the medium-induced radiation, or the kinematic constraint $k_{\perp}<\omega$, which are not properly included in the current formalism. Fortunately though, this theoretical uncertainty is not 
important for the angular distribution of the energy loss: the relevant curves in figure 12 are essentially flat for $x_{c} / x_{0} \gtrsim 1 / \bar{\alpha}^{2} \simeq 10$, i.e. for angles $\theta_{0} \gtrsim \theta_{s}$.

To conclude, let us try to make contact with the phenomenology of di-jet asymmetry at the LHC. As explained in the Introduction, this requires some care and additional assumptions, because the present formalism is not developed well enough to permit direct comparison with the data. In particular, by treating all the partons as being on-shell, we have neglected the 'vacuum-like' radiation, i.e. the parton fragmentation triggered by their virtuality. This mechanism is already effective for a jet fragmenting in the vacuum, in which case it is encompassed by the DGLAP equation. In the presence of a dense medium, both types of radiation - medium-induced and virtuality-triggered - are a priori expected and their interplay is generally complicated (and in any case beyond the scope of the present analysis). Note however that these two mechanism have rather different predictions for the energy transport to large angles. For the medium-induced radiation à la BDMPSZ, this proceeds via efficient multiple branchings leading to many soft quanta, as discussed at length in this paper. By contrast, for the vacuum-like radiations, this involves mainly three-jet (or multi-jet) events, as produced via hard branchings; then, the energy is carried to large angles by only few, but relatively hard, hadrons. The final states look quite different in the two cases (e.g. the medium-induced branchings lead to a larger multiplicity for soft hadrons) and such qualitative differences are indeed visible in the data [8]. Given this physical distinction and by lack of a better, unified, treatment of both mechanisms, we shall merely assume that the corresponding effects at large angles can be incoherently added to each other. It then makes sense to view our current formalism as describing the difference between the energy loss at large angles in nucleus-nucleus ('dense medium') versus proton-proton ('vacuum') collisions. This difference can in turn be estimated from the LHC data for di-jet asymmetry, albeit its extraction involves various assumptions and hence must be taken with care.

With this in mind, let us recall that a distinguished prediction of the turbulent mechanism for medium-induced fragmentation refers to variation of the jet energy with increasing the jet opening angle $\theta_{0}$, i.e. the function $E_{J}\left(\theta_{0}\right)$ introduced after eq. (4.9): as shown by the solid curve in figure 12, one expects a very slow rise of this function with $\theta_{0}$ and a significant 'offset' at very large angles $\theta_{0} \gtrsim \pi$. A recent analysis of di-jet asymmetry by CMS [8] has measured the energy imbalance between two nearly back-to-back jets as a function of the jet 'radius parameter' $R$ (essentially, our $\theta_{0}$ ), for both $\mathrm{p}+\mathrm{p}$ and $\mathrm{Pb}+\mathrm{Pb}$ collisions. This imbalance should decrease (in modulus) with increasing $R$ and approach zero after integrating over all angles $(R \approx 2 \pi)$, since there is no missing energy. And indeed the respective curves in figure 7 of ref. [8] show a rather strong dependence upon $R$, meaning a rapid recovery of the energy of the subleading jet with increasing $R\left(\approx \theta_{0}\right)$, for both $\mathrm{p}+\mathrm{p}$ and $\mathrm{Pb}+\mathrm{Pb}$; clearly, this measured $R$-dependence is much stronger than the one shown in figure 12. Yet, as previously explained, it is only the difference between these two experimental curves that can be associated with the medium-induced fragmentation and hence compared with our solid curve in figure 12. Remarkably, by inspection of figure 7 in ref. [8], one sees that this difference looks essentially flat in $R$ within the error bars and, moreover, it is quite large, in the ballpark of 5 to $10 \mathrm{GeV}$, for all angles up to $R \simeq 2$. This 
is in qualitative and even semi-quantitative agreement with figure 12, as we now explain.

For quantitative estimates, we shall use $\hat{q}=1 \mathrm{GeV}^{2} / \mathrm{fm}$ (an average value supported by recent phenomenological analyses [39]), $L=4 \mathrm{fm}$, and $\bar{\alpha}=0.3$. For these choices one finds $\omega_{c}=\hat{q} L^{2} / 2 \simeq 40 \mathrm{GeV}, \omega_{s}=\bar{\alpha}^{2} \omega_{c} \simeq 4 \mathrm{GeV}$, and $\theta_{c} \simeq 0.05$. This value for $\omega_{c}$ is significantly smaller than the typical jet energy $E \geq 100 \mathrm{GeV}$ in the experimental set-up for di-jet asymmetry at the LHC. Furthermore, the non-perturbative scale $\omega_{s}$ for the onset of multiple branching is reasonably hard for perturbation theory to apply; in particular, it is significantly harder than the medium 'temperature' $T \lesssim 1 \mathrm{GeV}$, as assumed in our previous developments. Finally, $\theta_{c} \simeq 0.05$ is much smaller than a typical jet radius parameter used in the phenomenology $(R \geq 0.2)$, meaning that the hard medium-induced emissions with $\omega \sim \omega_{c}$ do not contribute to the energy loss by the jet. The latter is rather controlled by very soft quanta with energies $\omega \lesssim \omega_{s}$, which are abundantly produced via multiple branchings and propagate at large angles $\theta \gtrsim \theta_{s}$, with $\theta_{s}=\theta_{c} / \bar{\alpha}^{2} \simeq 0.5$.

In this high-energy regime, the energy $\Delta E_{\text {flow }}$ carried away by the turbulent flow is quasi-independent of the original jet energy $E$ and can be estimated according to eq. (4.4):

$$
\Delta E_{\text {flow }} \simeq 0.32 \omega_{c} \simeq 13 \mathrm{GeV} .
$$

As anticipated, this value compares reasonably well with the corresponding experimental results $[2,8]$, notably with the value of the 'offset' at large angles in $\mathrm{Pb}+\mathrm{Pb}$ collisions, as read from figure 7 in ref. [8]. Albeit encouraging, this good agreement must be taken with care and further studies are certainly needed before being able to claim a successful phenomenology.

\section{Acknowledgments}

We are grateful to $\mathrm{Al}$ Mueller for insightful comments on the manuscript. We acknowledge useful related discussions with Jean-Paul Blaizot and Yacine Mehtar-Tani. This research is supported by the European Research Council under the Advanced Investigator Grant ERC-AD-267258. Figure 1 has been made with Jaxodraw [40].

\section{A Perturbation theory for the rate equation}

In this appendix, we shall discuss the perturbative solution to the rate equation with a source, eq. (3.8), as obtained via successive iterations of the branching term $\bar{\alpha} \mathcal{I}\left[D_{\text {rad }}\right]$ in the r.h.s. This is tantamount to an expansion in powers of $\bar{\alpha} \tau$ in which the source term $\mathcal{S}_{0}(x)=\bar{\alpha} / \sqrt{x}$ (including its factor $\bar{\alpha}$ ) is treated as a quantity of $\mathcal{O}(1)$. The zeroth order result is $D_{\text {rad }}^{(0)}=\bar{\alpha} \tau / \sqrt{x}$, while the first iteration, as obtained by evaluating the branching term $\bar{\alpha} \mathcal{I}\left[D_{\text {rad }}\right]$ with the zeroth order result and integrating over $\tau$, yields

$$
D_{\text {rad }}^{(1)}(x, \tau)=\frac{\bar{\alpha}^{2} \tau^{2}}{2} \int \mathrm{d} z \mathcal{K}(z)\left\{\Theta\left(z-\frac{x}{x_{c}}\right) \frac{z}{x}-\frac{z}{x}\right\}=-\frac{\bar{\alpha}^{2} \tau^{2}}{2 x} \int_{0}^{x / x_{c}} \mathrm{~d} z z \mathcal{K}(z) .
$$

The net result, which is negative, is due to an excess in the phase-space for the loss term, at $z<x / x_{c}$. To simplify the final integral over $z$, we shall restrict ourselves to the simplified 
kernel $\mathcal{K}_{0}(z)$. In that case, one can easily compute (say, by changing the integration variable as $z \equiv(u-1) / u)$

$$
\int_{0}^{x / x_{c}} \mathrm{~d} z z \mathcal{K}_{0}(z)=\int_{0}^{x / x_{c}} \frac{\mathrm{d} z}{\sqrt{z}(1-z)^{3 / 2}}=2 \sqrt{\frac{x}{x_{c}-x}} .
$$

When inserted into eq. (A.1), this confirms the result (3.22) for $D_{\text {rad }}^{(1)}$.

Note that the small $-x$ limit (in the sense that $x / x_{c} \ll 1$ ) of the result in eq. (A.2) would be the same for the full kernel $\mathcal{K}(z)$ : indeed, when $z<x / x_{c} \ll 1$, one can approximate $f(z) \simeq 1$ in eq. (2.6). This confirms that the limited expansion shown in eq. (3.44) holds for the physical kernel.

Returning to the simplified kernel $\mathcal{K}_{0}(z)$, in which case eq. (A.2) holds for any $x<x_{c}$, let us also compute the second iteration, by evaluating the branching term with the first order correction in eq. (3.22). One can write

$$
\begin{aligned}
\mathcal{I}\left[D_{\text {rad }}^{(1)}\right](x, \tau) & =-\frac{\bar{\alpha}^{2} \tau^{2}}{x} \int \mathrm{d} z z \mathcal{K}(z)\left\{\Theta\left(z-\frac{x}{x_{c}}\right) \frac{1}{\sqrt{x_{c}-x / z}}-\frac{1}{\sqrt{x_{c}-x}}\right\} \\
& =\frac{2 \bar{\alpha}^{2} \tau^{2}}{\sqrt{x}\left(x_{c}-x\right)}-\frac{\bar{\alpha}^{2} \tau^{2}}{x} \int_{x / x_{c}}^{1} \mathrm{~d} z z \mathcal{K}(z)\left\{\frac{1}{\sqrt{x_{c}-x / z}}-\frac{1}{\sqrt{x_{c}-x}}\right\} .
\end{aligned}
$$

Let us denote by $\mathcal{J}$ the integral in the second line above. After changing the integration variable according to $z=u /(u+1)$, this becomes

$$
\mathcal{J}=\int_{x / x_{c}}^{1} \mathrm{~d} z z \mathcal{K}(z)\left\{\frac{1}{\sqrt{x_{c}-x / z}}-\frac{1}{\sqrt{x_{c}-x}}\right\}=\frac{1}{\sqrt{x_{c}-x}} \int_{u_{0}}^{\infty} \mathrm{d} u\left\{\frac{1}{\sqrt{u-u_{0}}}-\frac{1}{\sqrt{u}}\right\}
$$

where we denoted $u_{0} \equiv x /\left(x_{c}-x\right)$. For any finite value of $u_{0}$, the above integral over $u$ is well defined and can be evaluated as

$$
\begin{aligned}
\mathcal{J} & =\frac{2}{\sqrt{x_{c}-x}} \lim _{u_{M} \rightarrow \infty}\left\{\sqrt{u_{M}-u_{0}}-\sqrt{u_{M}}+\sqrt{u_{0}}\right\} \\
& =\frac{2}{\sqrt{x_{c}-x}} \lim _{u_{M} \rightarrow \infty}\left\{\sqrt{u_{0}}-\frac{u_{0}}{2 \sqrt{u_{M}}}\right\}=\frac{2 \sqrt{x}}{x_{c}-x},
\end{aligned}
$$

where $u_{M}$ is a sharp upper cutoff on $u$ that has been introduced at intermediate steps in order to separate the two terms within the braces in the integral in eq. (A.4). When inserting the final result from eq. (A.5) into the second line of eq. (A.3), one finds that it precisely cancels the other term there, so that the net result of this second iteration is exactly zero: $\mathcal{I}\left[D_{\text {rad }}^{(1)}\right]=0$. Accordingly, the perturbative series becomes trivial (in the sense that all the higher order terms vanish) after the first iteration, and then the overall result is just the sum of the first two terms: $D_{\text {rad }}=D_{\text {rad }}^{(0)}+D_{\text {rad }}^{(1)}$. This is the result that has been announced towards the end of section 3.2.

Now, the fact that the function $D_{\text {rad }}^{(1)}(x, \tau)$ is an exact fixed point of the branching term is indeed correct and should not be a surprise: in section 3.3, we have seen that the very same function of $x$, namely $D_{\text {as }}(x) \propto 1 / \sqrt{x\left(x_{c}-x\right)}$, emerges as the exact solution to the 
rate equation for the case of a source localized at $x=x_{c}$ (cf. eq. (3.32)). Since the source vanishes at any $x<x_{c}$, this is tantamount to saying that $D_{\text {as }}(x)$ is an exact fixed point for the branching term: $\mathcal{I}\left[D_{\mathrm{as}}\right]=0$. This solution $D_{\text {as }}(x)$ becomes divergent when $x \rightarrow x_{c}$, but this is indeed a real property of that particular problem, because the respective source $\mathcal{S}(x)=A \delta\left(x-x_{c}\right)$ diverges at the end of the spectrum.

On the other hand, for the delocalized source $\mathcal{S}_{0}(x)=\bar{\alpha} / \sqrt{x}$, no such divergence is expected (as also confirmed by the exact manipulations in section 3.2), hence the iterative solution $D_{\text {rad }}=D_{\text {rad }}^{(0)}+D_{\text {rad }}^{(1)}$ cannot be fully right: it fails when $x \rightarrow x_{c}$. The mathematical reason for this failure can be traced to the subtlety of the limit $x \rightarrow x_{c}$ in relation with the manipulations in eqs. (A.4)-(A.5): clearly, these manipulations become ambiguous when $x \rightarrow x_{c}$, or $u_{0} \rightarrow \infty$, since this limit $u_{0} \rightarrow \infty$ does not commute with the limit $u_{M} \rightarrow \infty$.

Open Access. This article is distributed under the terms of the Creative Commons Attribution License (CC-BY 4.0), which permits any use, distribution and reproduction in any medium, provided the original author(s) and source are credited.

\section{References}

[1] ATLAS collaboration, Observation of a centrality-dependent dijet asymmetry in lead-lead collisions at $\sqrt{s_{N N}}=2.77$ TeV with the ATLAS detector at the LHC, Phys. Rev. Lett. 105 (2010) 252303 [arXiv: 1011.6182] [INSPIRE].

[2] CMS collaboration, Observation and studies of jet quenching in $\mathrm{PbPb}$ collisions at nucleon-nucleon center-of-mass energy $=2.76 \mathrm{TeV}$, Phys. Rev. C 84 (2011) 024906 [arXiv: 1102.1957] [INSPIRE].

[3] CMS collaboration, Jet momentum dependence of jet quenching in $\mathrm{PbPb}$ collisions at $\sqrt{s_{N N}}=2.76$ TeV, Phys. Lett. B 712 (2012) 176 [arXiv:1202.5022] [INSPIRE].

[4] ATLAS collaboration, Measurement of the jet radius and transverse momentum dependence of inclusive jet suppression in lead-lead collisions at $\sqrt{s_{N N}}=2.76 \mathrm{TeV}$ with the ATLAS detector, Phys. Lett. B 719 (2013) 220 [arXiv:1208.1967] [INSPIRE].

[5] CMS collaboration, Modification of jet shapes in PbPb collisions at $\sqrt{s_{N N}}=2.76 \mathrm{TeV}$, Phys. Lett. B 730 (2014) 243 [arXiv:1310.0878] [InSPIRE].

[6] CMS collaboration, Measurement of jet fragmentation in PbPb and $p p$ collisions at $\sqrt{s_{N N}}=2.76$ TeV, Phys. Rev. C 90 (2014) 024908 [arXiv:1406.0932] [InSPIRE].

[7] ATLAS collaboration, Measurement of inclusive jet charged-particle fragmentation functions in $\mathrm{Pb}+\mathrm{Pb}$ collisions at $\sqrt{s_{N N}}=2.76$ TeV with the ATLAS detector, Phys. Lett. $\mathbf{B} 739$ (2014) 320 [arXiv:1406.2979] [INSPIRE].

[8] CMS collaboration, Measurement of momentum flow relative to the dijet system in $\mathrm{PbPb}$ and pp collisions at $\sqrt{s_{N N}}=2.76 \mathrm{Te}$, CMS-PAS-HIN-14-010 (2014.

[9] Y. Mehtar-Tani, C.A. Salgado and K. Tywoniuk, Anti-angular ordering of gluon radiation in QCD media, Phys. Rev. Lett. 106 (2011) 122002 [arXiv: 1009.2965] [INSPIRE].

[10] Y. Mehtar-Tani, C.A. Salgado and K. Tywoniuk, Jets in QCD media: from color coherence to decoherence, Phys. Lett. B 707 (2012) 156 [arXiv:1102.4317] [InSPIRE]. 
[11] J. Casalderrey-Solana, J.G. Milhano and U.A. Wiedemann, Jet quenching via jet collimation, J. Phys. G 38 (2011) 035006 [arXiv:1012.0745] [InSPIRE].

[12] G.-Y. Qin and B. Müller, Explanation of di-jet asymmetry in $P b+P b$ collisions at the Large Hadron Collider, Phys. Rev. Lett. 106 (2011) 162302 [Erratum ibid. 108 (2012) 189904] [arXiv: 1012.5280] [INSPIRE].

[13] J. Casalderrey-Solana and E. Iancu, Interference effects in medium-induced gluon radiation, JHEP 08 (2011) 015 [arXiv: 1105.1760] [INSPIRE].

[14] J.-P. Blaizot, F. Dominguez, E. Iancu and Y. Mehtar-Tani, Medium-induced gluon branching, JHEP 01 (2013) 143 [arXiv: 1209.4585] [INSPIRE].

[15] J. Casalderrey-Solana, Y. Mehtar-Tani, C.A. Salgado and K. Tywoniuk, New picture of jet quenching dictated by color coherence, Phys. Lett. B 725 (2013) 357 [arXiv:1210.7765] [INSPIRE].

[16] J.-P. Blaizot, E. Iancu and Y. Mehtar-Tani, Medium-induced QCD cascade: democratic branching and wave turbulence, Phys. Rev. Lett. 111 (2013) 052001 [arXiv:1301.6102] [INSPIRE].

[17] J.-P. Blaizot, F. Dominguez, E. Iancu and Y. Mehtar-Tani, Probabilistic picture for medium-induced jet evolution, JHEP 06 (2014) 075 [arXiv:1311.5823] [INSPIRE].

[18] E. Iancu, Di-jet asymmetry and wave turbulence, arXiv:1404.4566 [INSPIRE].

[19] A. Kurkela and U.A. Wiedemann, Picturing perturbative parton cascades in QCD matter, Phys. Lett. B 740 (2015) 172 [arXiv:1407.0293] [InSPIRE].

[20] J.-P. Blaizot, Y. Mehtar-Tani and M.A.C. Torres, Angular structure of the in-medium QCD cascade, arXiv:1407.0326 [INSPIRE].

[21] L. Apolinário, N. Armesto, J.G. Milhano and C.A. Salgado, Medium-induced gluon radiation and colour decoherence beyond the soft approximation, JHEP 02 (2015) 119 [arXiv: 1407.0599] [INSPIRE].

[22] R. Baier, A.H. Mueller, D. Schiff and D.T. Son, 'Bottom up' thermalization in heavy ion collisions, Phys. Lett. B 502 (2001) 51 [hep-ph/0009237] [INSPIRE].

[23] S. Jeon and G.D. Moore, Energy loss of leading partons in a thermal QCD medium, Phys. Rev. C 71 (2005) 034901 [hep-ph/0309332] [INSPIRE].

[24] B. Schenke, C. Gale and S. Jeon, MARTINI: an event generator for relativistic heavy-ion collisions, Phys. Rev. C 80 (2009) 054913 [arXiv: 0909. 2037] [INSPIRE].

[25] R. Baier, Y.L. Dokshitzer, A.H. Mueller, S. Peigne and D. Schiff, Radiative energy loss of high-energy quarks and gluons in a finite volume quark-gluon plasma, Nucl. Phys. B 483 (1997) 291 [hep-ph/9607355] [INSPIRE].

[26] R. Baier, Y.L. Dokshitzer, A.H. Mueller, S. Peigne and D. Schiff, Radiative energy loss and $p_{T}$ broadening of high-energy partons in nuclei, Nucl. Phys. B 484 (1997) 265 [hep-ph/9608322] [INSPIRE].

[27] B.G. Zakharov, Fully quantum treatment of the Landau-Pomeranchuk-Migdal effect in QED and QCD, JETP Lett. 63 (1996) 952 [hep-ph/9607440] [INSPIRE].

[28] B.G. Zakharov, Radiative energy loss of high-energy quarks in finite size nuclear matter and quark-gluon plasma, JETP Lett. 65 (1997) 615 [hep-ph/9704255] [INSPIRE]. 
[29] R. Baier, Y.L. Dokshitzer, A.H. Mueller and D. Schiff, Medium induced radiative energy loss: equivalence between the BDMPS and Zakharov formalisms, Nucl. Phys. B 531 (1998) 403 [hep-ph/9804212] [INSPIRE].

[30] V. Zakharov, V. Lvov and G. Falkovich, Kolmogorov spectra of turbulence, volume 1, Springer, Germany (1992).

[31] S. Nazarenko, Wave turbulence, Springer, Germany (2011).

[32] A. Kurkela and G.D. Moore, Thermalization in weakly coupled nonabelian plasmas, JHEP 12 (2011) 044 [arXiv: 1107.5050] [INSPIRE].

[33] T. Liou, A.H. Mueller and B. Wu, Radiative $p_{\perp}$-broadening of high-energy quarks and gluons in QCD matter, Nucl. Phys. A 916 (2013) 102 [arXiv:1304.7677] [INSPIRE].

[34] E. Iancu, The non-linear evolution of jet quenching, JHEP 1410 (2014) 95 [arXiv: 1403.1996] [INSPIRE].

[35] J.-P. Blaizot and Y. Mehtar-Tani, Renormalization of the jet-quenching parameter, Nucl. Phys. A 929 (2014) 202 [arXiv:1403.2323] [INSPIRE].

[36] E. Iancu and D.N. Triantafyllopoulos, Running coupling effects in the evolution of jet quenching, Phys. Rev. D 90 (2014) 074002 [arXiv:1405.3525] [INSPIRE].

[37] $\mathrm{B} . \mathrm{Wu}$, Radiative energy loss and radiative $p_{\perp}$-broadening of high-energy partons in $Q C D$ matter, JHEP 12 (2014) 081 [arXiv: 1408.5459] [INSPIRE].

[38] R. Baier, Y.L. Dokshitzer, A.H. Mueller and D. Schiff, Quenching of hadron spectra in media, JHEP 09 (2001) 033 [hep-ph/0106347] [INSPIRE].

[39] JET collaboration, K.M. Burke et al., Extracting the jet transport coefficient from jet quenching in high-energy heavy-ion collisions, Phys. Rev. C 90 (2014) 014909 [arXiv: 1312.5003] [INSPIRE].

[40] D. Binosi and L. Theussl, JaxoDraw: a graphical user interface for drawing Feynman diagrams, Comput. Phys. Commun. 161 (2004) 76 [hep-ph/0309015] [INSPIRE]. 